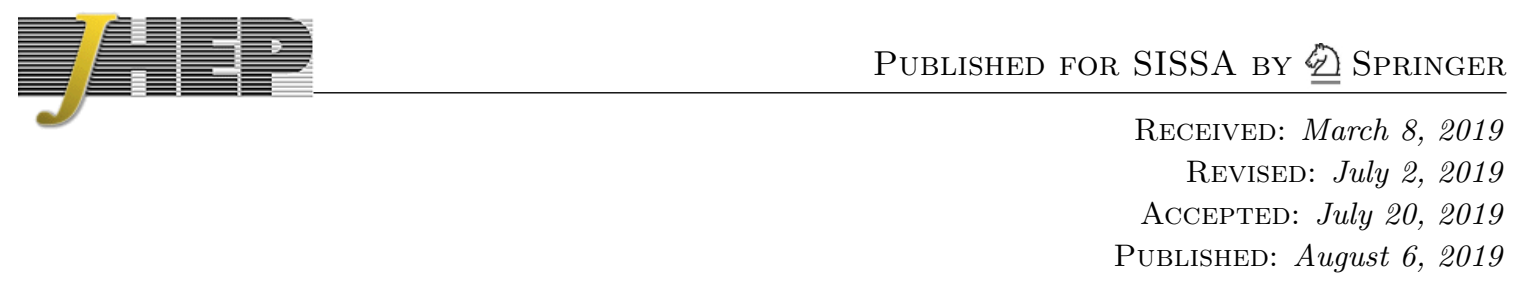

\title{
Supersymmetric inflation from the fifth dimension
}

\section{Kaustubh Deshpande and Raman Sundrum}

Maryland Center for Fundamental Physics, Department of Physics, University of Maryland, College Park, MD 20742, U.S.A.

E-mail: ksd@umd.edu, raman@umd.edu

ABSTRACT: We develop a supersymmetric bi-axion model of high-scale inflation coupled to supergravity, in which the axionic structure originates from, and is protected by, gauge symmetry in an extra dimension. While local supersymmetry (SUSY) is necessarily Higgsed at high scales during inflation we show that it can naturally survive down to the $\sim \mathrm{TeV}$ scale in the current era in order to resolve the electroweak hierarchy problem. We show how a suitable inflationary effective potential for the axions can be generated at tree-level by charged fields under the higher-dimensional gauge symmetry. The inflationary trajectory lies along the lightest direction in the bi-axion field space, with periodic effective potential and an effective super-Planckian field range emerging from fundamentally sub-Planckian dynamics. The heavier direction in the field space is shown to also play an important role, as the dominant source of super-Higgsing during inflation. This model presents an interesting interplay of tuning considerations relating the electroweak hierarchy, cosmological constant and inflationary superpotential, where maximal naturalness favors SUSY breaking near the electroweak scale after inflation. The scalar superpartner of the axionic inflaton, the "sinflaton", can naturally have Hubble mass during inflation and sufficiently strong coupling to the inflaton to mediate primordial non-Gaussianities of observable strength in future 21-cm surveys. Non-minimal charged fields under the higher-dimensional gauge symmetry can contribute to periodic modulations in the CMB, within the sensitivity of ongoing measurements.

Keywords: Cosmology of Theories beyond the SM, Field Theories in Higher Dimensions, Supersymmetric Gauge Theory

ARXIV EPRINT: 1902.05559 


\section{Contents}

1 Introduction 1

2 The Kallosh-Linde-Rube model $\quad 4$

$\begin{array}{lll}3 & \text { SUSY bi-axion model } & 7\end{array}$

$\begin{array}{lll}3.1 & \text { Light axion supermultiplet from 5D SUSY gauge theory } & 7\end{array}$

$\begin{array}{lll}3.2 & \text { Bi-axion generalization to realize } f_{\text {eff }}>M_{\mathrm{Pl}} & 10\end{array}$

$\begin{array}{ll}3.3 & \text { Adding SUGRA and identifying the SUSY vacuum }\end{array}$

4 Inflationary history $\quad 13$

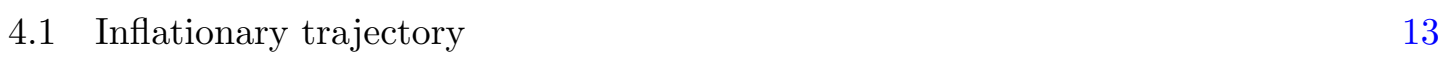

$\begin{array}{lll}\text { 4.2 SUSY breaking during inflation } & 15\end{array}$

$\begin{array}{lll}4.3 & \text { SUSY breaking after inflation } & 16\end{array}$

4.4 Interplay of electroweak, cosmological constant and superpotential tunings $\quad 17$

5 Observable signals $\quad 18$

$\begin{array}{lll}5.1 & \text { Primordial non-Gaussianities } & 19\end{array}$

$\begin{array}{lll}5.1 .1 & \text { Sinflaton } & 19\end{array}$

$\begin{array}{ll}\text { 5.1.2 Boundary-localized gauge singlets } & 21\end{array}$

$\begin{array}{ll}5.2 & \text { Periodic modulations in the CMB }\end{array}$

6 Conclusions $\quad 22$

$\begin{array}{ll}\text { A SUGRA preliminaries } & 23\end{array}$

\section{Introduction}

Cosmic inflation provides an attractive framework for understanding the robustness of the early state of our universe (see [1] for a review). Its simplest implementation driven by a slowly rolling scalar field (inflaton) requires a very flat inflaton potential, suggesting that the inflaton is a pseudo-Goldstone boson of a spontaneously broken global symmetry. A small explicit breaking of the symmetry can then give rise to a weak potential naturally varying on the scale of the spontaneous breaking, $f$. A canonical example is given by the model of "Natural Inflation" [2], with periodic inflaton potential,

$$
V(\phi)=V_{0}\left(1-\cos \frac{\phi}{f}\right) .
$$


However, even a crude fit to the Cosmic Microwave Background (CMB) data [3] requires $f \gtrsim M_{\mathrm{Pl}},{ }^{1}$ which conflicts with our general expectation that there should be no dynamical scales above the Planck scale, and with the particular arguments that global symmetries themselves are ill-defined in the context of Quantum Gravity [5-7].

These concerns can be resolved by (a) relating but not identifying the scale over which the inflaton potential varies with the scale of spontaneous symmetry breaking, and (b) realizing the spontaneously broken approximate symmetries as accidental symmetries in the IR rather than fundamental global symmetries in the UV. The simplest version of (a) is given by beginning with two pseudo-Goldstone bosons, $\phi_{A}$ and $\phi_{B}$, for two global symmetries $\mathrm{U}(1)_{A} \times \mathrm{U}(1)_{B}$ spontaneously broken at approximately the same scale $f_{A}, f_{B} \approx$ $f \ll M_{\mathrm{Pl}}[8]$. For suitable explicit symmetry breaking sources one can then generate a potential of the form

$$
V\left(\phi_{A}, \phi_{B}\right)=V_{0}^{(1)}\left(1-\cos \frac{\phi_{B}}{f_{B}}\right)+V_{0}^{(2)}\left[1-\cos \left(\frac{\phi_{A}}{f_{A}}+N \frac{\phi_{B}}{f_{B}}\right)\right],
$$

where $N$ represents a large charge under $\mathrm{U}(1)_{B}$ for one of the "spurions" characterizing the explicit breaking. Naively, this makes the problem worse, since the potential varies in the $\phi_{A}$ direction on the scale $f \ll M_{\mathrm{Pl}}$, and in the $\phi_{B}$ direction on the scale $f / N \ll f$, while CMB data suggests a potential varying more slowly than the Planck scale. However, just such a potential can arise when we properly consider the mass eigenstates. Taking for simplicity $V_{0}^{(1),(2)} \approx V_{0}$, these are given by heavy and light directions in field space,

$$
\phi_{h} \equiv \phi_{B}+\frac{1}{N} \phi_{A}, \phi_{l} \equiv \phi_{A}-\frac{1}{N} \phi_{B}
$$

After setting the heavy $\phi_{h}$ to its vacuum expectation value (VEV), we can obtain the effective potential for the light field $\phi_{l}$ as

$$
\left.V_{\mathrm{eff}}\left(\phi_{l}\right)\right|_{\left\langle\phi_{h}\right\rangle \approx 0} \approx V_{0}\left(1-\cos \frac{\phi_{l}}{N f}\right) .
$$

This corresponds to an effective Natural Inflation model, with inflaton $\phi_{l}$ and an emergent scale of potential variation $f_{\text {eff }}=N f$, which can be $>M_{\mathrm{Pl}}$ even though $f<M_{\mathrm{Pl}}$, for sufficiently large spurious charge $N$. We will refer to this as the "Bi-axion inflation" model.

An attractive microscopic realization of Bi-axion inflation satisfying (b), based on the mechanism of "extranatural inflation" [9], is provided by using gauge symmetry in an extra dimension [10]. If the higher-dimensional spacetime is highly warped so as to have an $\mathrm{AdS}_{5} / \mathrm{CFT}_{4}$ type holographic purely-4D dual description, then the dual interpretation is that the axions are composite Goldstone bosons of some strong dynamics (see e.g. [11]), analogous to the pions of QCD, and the spontaneously broken symmetries are accidental or emergent symmetries below the Planck scale. Here, we just briefly summarize the unwarped (or mildly warped) higher-dimensional case. The 4D axions above are realized

\footnotetext{
${ }^{1}$ This is an example of the model-independent Lyth bound [4] in the case of Natural Inflation model.
} 
as gauge-invariant Wilson-loops (or lines, given suitable boundary conditions) around (or across) the compact extra dimension,

$$
\phi_{A} \equiv \int_{0}^{L} A_{5} d x_{5}, \phi_{B} \equiv \int_{0}^{L} B_{5} d x_{5}
$$

Charged matter propagating in the $5 \mathrm{D}$ bulk, $H_{1}$ and $H_{2}$, with mass $m$, can generate the potential (1.2) for $\phi_{A}$ and $\phi_{B}$, given that they are charged under the two gauge groups as $(0,1)$ and $(1, N)$, respectively. The scales $f_{A}, f_{B}$ emerge as

$$
f_{A}=\frac{1}{g_{A} L}, f_{B}=\frac{1}{g_{B} L} .
$$

The potential in (1.2) can be generated minimally by the loop contributions of $H_{1}, H_{2}$ via the "Hosotani mechanism" [12] which gives

$$
V_{0}^{\text {loop }} \sim \frac{e^{-m L}}{L^{4}}
$$

in (1.4), as well as "higher harmonics" accompanied by higher powers of $e^{-m L}$. As studied in [13], bi-axion extranatural inflation can also non-trivially satisfy the plausible constraints of the Weak Gravity Conjecture (WGC) [14]. These quantum gravity constraints are an even stronger form of the arguments forbidding fundamental global symmetries, to also forbid UV gauge symmetries with very weak gauge couplings (relative to gravitational strength). These higher dimensional realizations of bi-axion inflation can be generalized to multiple-axion models, which then allow for more modest values of charge, $N[10,13]$.

In this paper, we study compatibility of the bi-axion inflation scenario arising from higher dimensional gauge theory with the scenario of $\sim \mathrm{TeV}$-scale supersymmetry (SUSY) for resolving the electroweak hierarchy problem. In the presence of SUSY, the loop contributions from the charged matter fields to the effective potential of $4 \mathrm{D}$ axions cancel out. We are hence forced to have tree-level contributions for the same, which can be achieved if $H_{1}, H_{2}$ have non-zero VEVs $\left(v, v^{\prime}\right)$ at both the boundaries, which generates

$$
V_{0}^{\text {tree }} \sim e^{-m L} m v v^{\prime} .
$$

Obviously, the question of whether the above-mentioned very plausible and robust forms of inflation are naturally realizable within the constraints of supergravity (SUGRA) dynamics in the UV, with SUSY being present at $\sim$ collider energies today, is of considerable importance to our picture of fundamental physics and the prospects for experiments and observations. See [15-21] for other discussions of bi-axion inflation combined with SUSY, where the axions have alternative UV realizations. See [22-28] for other attempts to reconcile low energy SUSY and inflation from a UV perspective. We will also explore the possible new signatures from extra fields in the axion supermultiplets, most notably in the form of primordial non-Gaussianities (NG) in the cosmological collider physics program [29-39] as well as periodic modulations in the CMB [13, 40-48].

Models of single-field inflation with relatively simple potentials, such as Natural Inflation and its variants, necessarily operate at high scales in order to satisfy cosmological 
data, with inflationary Hubble scale $H_{\text {inf }} \sim 10^{13}-10^{14} \mathrm{GeV}$. The recently released Planck 2018 data places tight constraints on such high-scale models, especially given the nonobservation of CMB B-modes induced by super-horizon gravitational waves [49]. Natural Inflation itself is now disfavored at $95 \%$ confidence level, but not ruled out. However, the bi-axionic structure of inflation from extra-dimensional gauge symmetry can generically produce multiple periodic terms in the potential (1.1), which can alleviate the tension above with a suitable and plausibly not very fine-tuned choice of parameters. We leave such a detailed analysis and appraisal for a future study. Furthermore, there are various ways discussed in the existing literature to relax these constraints for axion-based inflation, e.g. by realizing the structure of hybrid inflation from a bi-axion potential [50].

The paper is organized as follows. In section 2, we review a SUGRA-based inflation model, the "Kallosh-Linde-Rube model" [51, 52], which has many common features with our SUSY bi-axion model as developed in sections 3 and 4. In section 3, starting from the 5D SUSY gauge structure, we first construct a 4D effective theory of an axion supermultiplet. After generalizing it to the case of two axions, we account for (effective) 4D SUGRA couplings below the compactification scale. In section 4, we discuss the inflationary trajectory along the lightest direction in the field space with an effective super-Planckian field range and periodic potential, also stabilized along all the other heavier directions. We then describe the picture of SUSY breaking (SUSY) during inflation which we find to be caused mostly by the heavy sector and not the inflaton sector. Furthermore, we also account for the post-inflationary SUSY vacuum that we occupy today, which we find not affecting the inflationary dynamics significantly as long as the SUSY scale is much below the inflationary energy scale. This model presents an interesting interplay of fine-tunings in the electroweak (EW) sector, cosmological constant (CC), and superpotential which are connected together after incorporating the SUSY today. The superpotential fine-tuning favors SUSY at high-scale, however the net fine-tuning, dominated by the EW and CC fine-tunings, can be shown to favor SUSY at low-scale i.e. somewhat above the EW scale. In section 5, we discuss observable signals in the form of primordial NG and periodic modulations in the CMB. The "sinflaton", the real scalar partner of inflaton, can have $\mathcal{O}\left(H_{\text {inf }}\right)$ mass during inflation and sufficiently strong coupling to the inflaton to mediate primordial $\mathrm{NG}$ of observable strength in future experiments. A boundary-localized gauge singlet, in the presence of a shift-symmetric Kähler coupling, can also mediate sizeable primordial NG. Charged matter much heavier than the compactification scale, even only modestly below the 5D gauge theory cut-off, can contribute to periodic modulations in the CMB, within the sensitivity of ongoing searches. We conclude in section 6 .

We use units with the reduced Planck mass $M_{\mathrm{Pl}}=1$ everywhere in the paper, except sections 4.4 and 5, where we explicitly write factors of $M_{\mathrm{Pl}}$ in order to get a better sense of the numbers.

\section{The Kallosh-Linde-Rube model}

We seek a locally supersymmetric description of high-scale inflation in which SUSY is only broken somewhat above the weak scale today. Since the weak scale is $\ll H_{\text {inf }}$, we can first 
consider the supersymmetric limit of the ground state today. On the other hand, during inflation we know that the approximate de Sitter geometry is incompatible with SUSY. So inflation must be a spontaneous breaking (super-Higgsing) of SUSY within an excitation on top of today's SUSY vacuum, which we can also approximate to have zero vacuum energy (cosmological constant).

In order to have a light inflaton $(\phi)$, we will have an inflaton supermultiplet $(\Phi)$ with approximate shift symmetry. This can be implemented with $K(\Phi, \bar{\Phi})=K(\Phi+\bar{\Phi})$ and $\phi=\operatorname{Im}(\Phi)$. A small explicit breaking of the shift symmetry from the superpotential can generate slow-roll potential for $\phi$. Thus, the lightness of inflaton can be explained by its pseudo-Goldstone boson nature. However, implementing inflation with only this single supermultiplet is challenging [53]. In this case, the Goldstino of spontaneous SUSY during inflation would have to be the inflatino (then "eaten" by the gravitino). Consider $K=\frac{1}{2}(\Phi+\bar{\Phi})^{2}$ and $W=f(\Phi)$. Then, restricting to polynomial $f(\Phi)$ for illustration, in SUGRA, $V(\phi) \approx f^{\prime 2}(\phi / \sqrt{2})-3 f^{2}(\phi / \sqrt{2})$, which has a clear instability. ${ }^{2}$ This can be avoided by introducing a separate supermultiplet for the Goldstino during inflation.

We see that the Goldstino multiplet must be part of a sector that Higgses SUSY during inflation. One of the simplest models to describe spontaneous SUSY coupled to SUGRA, is the Polonyi model [57]:

$$
K=\bar{S} S-\lambda(\bar{S} S)^{2}, W=\mu S,
$$

with the addition of the non-minimal Kähler coupling $\lambda$. The SUSY order parameter in the vacuum is $\left.D_{S} W\right|_{\langle S\rangle \approx 0} \approx \mu \neq 0$. Spontaneous SUSY in this model gives rise to a massless Goldstino which however is eaten by the gravitino which then becomes massive ("super-Higgs mechanism"). The quartic term in the Kähler potential also makes the scalar heavy, with $m_{S}^{2} \approx 4 \lambda \mu^{2}$. Thus, there is no light particle in this sector. During inflation, in the limit of the slow-roll approximation i.e. for a fixed value of inflaton, the physics can be approximately described by this model. But we need to have a coupling between this sector $(S)$ and the inflaton $(\Phi)$ such that there is no SUSY at $\Phi=0$ (i.e. at the vacuum today) but with SUSY at $\Phi=\Phi_{0} \neq 0$ (i.e. during inflation). In other words, the $\mu$ parameter of (2.1) needs to be made $\Phi$-dependent in a suitable manner. This can be achieved with the following model $[51,52]$ :

$$
K=\frac{1}{2}(\Phi+\bar{\Phi})^{2}+\bar{S} S-\lambda(\bar{S} S)^{2}, W=S f(\Phi),
$$

which we will refer to as the "Kallosh-Linde-Rube (KLR) model" and consider as a toy model for our SUSY bi-axion model. All scalars except for $\phi=\operatorname{Im}(\Phi)$ can be shown to be heavy and thus the inflationary potential (see appendix A for SUGRA scalar potential) can be obtained as,

$$
\begin{aligned}
\left.V(\phi)\right|_{\langle S\rangle,\langle\eta\rangle} & =f^{2}(\phi / \sqrt{2}) \\
V_{\mathrm{inf}} & =3 H_{\mathrm{inf}}^{2}=f^{2}\left(\phi_{0} / \sqrt{2}\right) .
\end{aligned}
$$

\footnotetext{
${ }^{2}$ However, see [54-56] for attempts towards building "sGoldstino inflation" model.
} 
The SUSY order parameters for $\Phi$ and $S$ during inflation can be evaluated as follows:

$$
\left.D_{\Phi} W\right|_{\mathrm{inf}} \approx 0,\left.D_{S} W\right|_{\mathrm{inf}} \approx f\left(\Phi_{0}\right) \neq 0
$$

This implies that, as expected, SUSY during inflation is caused by the heavy sector $(S)$. Hence the Goldstino during inflation (further eaten by the gravitino) is equal to the fermion from the $S$-sector $\left(\psi_{S}\right)$ and not the inflatino $\left(\psi_{\Phi}\right)$.

The real scalar partner of the inflaton i.e. sinflaton $(\eta=\operatorname{Re}(\Phi))$, has the following mass coming from its coupling to the SUSY-breaking curvature from (2.2):

$$
m_{\eta} \approx \sqrt{6} H_{\mathrm{inf}} .
$$

This is within the favorable range of masses for observing it in primordial NG in the cosmological collider physics program. However, such a light sinflaton (i.e. $m_{\eta} \sim \mathcal{O}\left(H_{\text {inf }}\right)$ ) is not guaranteed from this class of models. Indeed, a higher order term in the Kähler potential with a direct coupling between $S$ and $\Phi$, respecting the shift symmetry of $\phi$,

$$
K \ni-\frac{c}{\Lambda^{2}}(\Phi+\bar{\Phi})^{2} \bar{S} S
$$

can give a large contribution to the sinflaton mass:

$$
m_{\eta}^{2} \approx 2 V_{\mathrm{inf}}+c \frac{V_{\mathrm{inf}}}{\Lambda^{2}} \approx 6 H_{\mathrm{inf}}^{2}\left(1+\frac{c}{2 \Lambda^{2}}\right)
$$

Thus, $m_{\eta} \sim \mathcal{O}\left(H_{\text {inf }}\right)$ for $\Lambda \approx \mathcal{O}(1) M_{\mathrm{Pl}}$. But, $m_{\eta} \gg H_{\text {inf }}$ is also possible with $\Lambda \ll M_{\mathrm{Pl}}$.

Even assuming $m_{\eta} \sim \mathcal{O}\left(H_{\mathrm{inf}}\right)$, in order for $\eta$ to mediate observable primordial NG, there has to be sufficiently strong coupling between it and the inflaton $(\phi)$. The SUGRA scalar potential from (2.2) does have such couplings, but these are shift-symmetry violating and hence very small, e.g. $\mathcal{L} \ni m_{\phi}^{2} \eta^{2} \phi^{2} \sim 10^{-10} \eta^{2} \phi^{2}$. However, higher order shift-symmetric terms in Kähler potential,

$$
K \ni \frac{c^{\prime}}{\Lambda^{2}}(\Phi+\bar{\Phi})^{4}
$$

can generate derivative-interactions as

$$
\mathcal{L} \ni \frac{c^{\prime}}{\Lambda^{2}}(\partial \phi)^{2} \eta^{2}
$$

This sinflaton-inflaton interaction (with a non-zero VEV for $\eta$ ) along with $m_{\eta} \sim \mathcal{O}\left(H_{\text {inf }}\right.$ ) can give rise to observable NG for sufficiently small $\Lambda$ and large $\langle\eta\rangle$. However, in this paper, we will not pursue the phenomenology of this model further.

The main drawback of this construction is that the origin of such a form of Lagrangian (2.2) is not explained within the model. Also, it suffers from the issue of transPlanckian field displacement needed for $\phi$, since a typical choice of $f(\Phi)$ in $(2.2)$ gives a large-field inflation model subject to the Lyth bound [4]. 


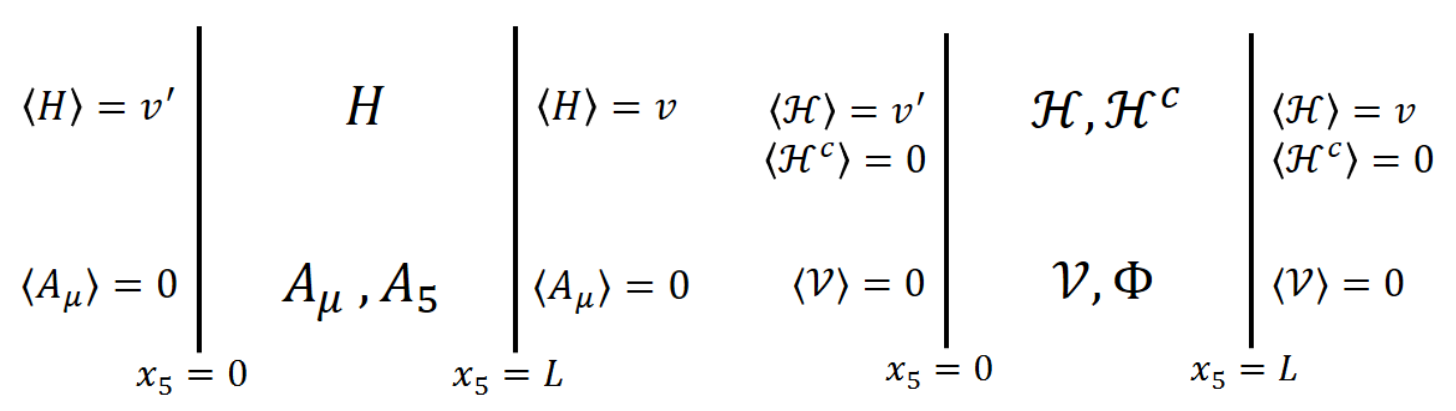

(a) Non-SUSY.

(b) SUSY.

Figure 1. 5D gauge field and charged matter: (a) non-SUSY and (b) SUSY version. See text and table 1 for details.

\section{SUSY bi-axion model}

In this section, we develop the setup of supersymmetric inflation with the pseudo-Goldstone boson (or axion) nature of inflaton derived from a gauge symmetry in a compact extra dimension ("extranatural inflation" [9]). Firstly, we describe how we obtain the effective theory of a light axion supermultiplet starting from the $\mathcal{N}=15 \mathrm{D}$ SUSY gauge theory. Then, we describe how to introduce two such axion supermultiplets in order to get $f_{\text {eff }}>$ $M_{\mathrm{Pl}}$ (for trans-Planckian field displacement satisfying the WGC). Finally, we also discuss how to take into account gravity, thus constructing our "SUSY bi-axion model".

As we will see later, this model has many common features with the KLR model described in section 2. It however provides a more UV-complete and robust picture of inflationary dynamics where the central features are governed by the 5D SUSY gauge theory structure.

\subsection{Light axion supermultiplet from 5D SUSY gauge theory}

In this sub-section, we will show how a single light axion supermultiplet can emerge from 5D SUSY gauge theory. The extension to the more realistic case of two axion supermultiplets follows in the next sub-section. Consider a flat extra dimension with boundaries, with a gauge field $A_{M}$ and a charged scalar field $H$ propagating in the bulk (see figure 1a). If $A_{\mu}$ and $A_{5}$ have, respectively, Dirichlet and Neumann boundary conditions at both the boundaries, then only $A_{5}$ has a zero-mode $\left(A_{5}^{(0)}\right)$. As mentioned in section 1 , if $H$ has nonzero VEVs at both the boundaries, then it gives a tree-level contribution to the effective potential of $A_{5}^{(0)}$.

Now, consider the full $5 \mathrm{D}$ supersymmetric version of this setup (see figure $1 \mathrm{~b}$ ). $\mathcal{N}=1$ 5 D SUSY is equivalent to $\mathcal{N}=24$ D SUSY which can be written in the $\mathcal{N}=14 \mathrm{D}$ SUSY language as follows [58] (see table 1). 5D SUSY gauge multiplet has a gauge field $\left(A_{M}\right)$, Dirac gaugino $\left(\chi_{\text {Dirac }}\right)$ and a real scalar $(\eta)$. These can be represented in $\mathcal{N}=14 \mathrm{D}$ SUSY language in terms of vector superfields $\mathcal{V}\left(x_{5}\right) \ni A_{\mu}\left(x_{5}\right), \chi_{1}\left(x_{5}\right)$ and chiral superfields $\Phi\left(x_{5}\right) \ni \eta\left(x_{5}\right)+i A_{5}\left(x_{5}\right), \chi_{2}\left(x_{5}\right)$, where the extra-dimensional coordinate $x_{5}$ is viewed as a mere continuous "label" from the $\mathcal{N}=14 \mathrm{D}$ viewpoint. Charged matter fields in 5D SUSY are part of a hypermultiplet which includes two complex scalars which are conjugates 


\begin{tabular}{|c|c|c|}
\hline 5D super-multiplet & 5 D fields & $\mathcal{N}=14$ D superfields \\
\hline Gauge multiplet & $A_{M}, \chi_{\text {Dirac }}, \eta_{\text {real }}$ & $\begin{array}{c}\text { Vector superfields: } \mathcal{V}\left(x_{5}\right) \ni A_{\mu}\left(x_{5}\right), \chi_{1}\left(x_{5}\right) \\
\text { Chiral superfields: } \Phi\left(x_{5}\right) \ni \eta\left(x_{5}\right)+i A_{5}\left(x_{5}\right), \chi_{2}\left(x_{5}\right)\end{array}$ \\
\hline Hypermultiplet & $H, H^{c}, \psi_{\text {Dirac }}$ & $\begin{array}{r}\text { Chiral superfields: } \mathcal{H}\left(x_{5}\right) \ni H\left(x_{5}\right), \psi\left(x_{5}\right) \\
\mathcal{H}^{c}\left(x_{5}\right) \ni H^{c}\left(x_{5}\right), \psi^{c}\left(x_{5}\right)\end{array}$ \\
\hline
\end{tabular}

Table 1. $\mathcal{N}=15 \mathrm{D}$ SUSY in the $\mathcal{N}=14 \mathrm{D}$ SUSY language.

of each other under the respective gauge group $\left(H, H^{c}\right)$ and a Dirac fermion $\left(\psi_{\text {Dirac }}\right)$. These can be represented in $\mathcal{N}=14$ D SUSY language in terms of chiral superfields with conjugate representations: $\mathcal{H}\left(x_{5}\right) \ni H\left(x_{5}\right), \psi\left(x_{5}\right) ; \mathcal{H}^{c}\left(x_{5}\right) \ni H^{c}\left(x_{5}\right), \psi^{c}\left(x_{5}\right)$, again with the continuous "label" $x_{5}$.

As illustrated in [58], imposing 4D SUSY and 5D Poincare symmetry automatically generates an emergent 5D SUSY. Thus, the full 5D Lorentz-invariant, gauge-invariant and SUSY action for a gauge multiplet and a charged hypermultiplet, keeping manifest only the $\mathcal{N}=14 \mathrm{D}$ SUSY, can be written as follows:

$$
\begin{aligned}
\mathcal{S}_{5}= & \int d^{4} x \int_{0}^{L} d x_{5}\left[\int d^{2} \theta \frac{1}{4} \mathcal{W}_{\alpha}^{2}+\text { h.c. }+\int d^{4} \theta\left\{\partial_{5} \mathcal{V}-\frac{1}{\sqrt{2}}(\Phi+\bar{\Phi})\right\}^{2}\right. \\
& \left.+\int d^{4} \theta\left(\mathcal{H}^{c} e^{g_{5}} \mathcal{V} \overline{\mathcal{H}}^{c}+\overline{\mathcal{H}} e^{-g_{5} \mathcal{V}} \mathcal{H}\right)+\int d^{2} \theta\left\{\mathcal{H}^{c}\left(m+\partial_{5}-\frac{g_{5}}{\sqrt{2}} \Phi\right) \mathcal{H}\right\}+\text { h.c. }\right] .
\end{aligned}
$$

As mentioned in section 1, in the presence of SUSY, we need tree-level contributions from charged matter to the effective potential of $A_{5}$, which can be achieved by the charged matter taking non-zero VEVs at the boundaries. Such VEVs break gauge invariance, but this is allowed because we have already broken gauge invariance by the Dirichlet boundary conditions for the boundary components of the gauge fields. These VEVs can be achieved by adding the following boundary-localized superpotential terms to the action:

$$
\delta \mathcal{S}_{5}=\int d^{4} x \int_{0}^{L} d x_{5}\left[\int d^{2} \theta\left\{\lambda(\mathcal{H}-v)^{2} \delta\left(x_{5}\right)+\lambda^{\prime}\left(\mathcal{H}-v^{\prime}\right)^{2} \delta\left(x_{5}-L\right)\right\}+\text { h.c. }\right] .
$$

Consider Dirichlet boundary conditions for $\mathcal{V}$ and $\mathcal{H}^{c}$ and Neumann boundary conditions for $\Phi$ and $\mathcal{H}$, at both the boundaries. We implement these boundary conditions via realizing the extra dimension with an interval as an "orbifold" of the circle. With the angular coordinate $(\theta)$ on the circle going from $-\pi$ to $\pi$, we identify the points $\theta$ with $-\theta$. Thus, half of the circumference of the extra-dimensional circle is the physical interval with $x_{5}$ going from 0 to $\pi R \equiv L$, where $R$ is the radius of the circle. The Dirichlet and Neumann boundary conditions for the fields in an interval can be implemented by assigning, respectively, odd and even parity under orbifold $(\theta \rightarrow-\theta)$. (See [59] for a review of this.)

Let us solve for the classical potential of this model. We need to integrate out the heavy fields (i.e. $\mathcal{H}, \mathcal{H}^{c}$ and the KK modes in $\mathcal{V}$ ) at tree-level to get an effective theory in terms of 
$\Phi$. We search for a supersymmetric vacuum of the full theory where inflation happens at an excited state with SUSY vacuum energy $V_{\text {inf }}$. Considering the inflationary energy scale to be much less than the masses of the heavy fields $\left(V_{\text {inf }}^{1 / 4} \ll m_{K K}, m\right),{ }^{3}$ to the leading order in $\frac{V_{\text {inf }}^{1 / 4}}{m_{K K}}$, for the purpose of the dynamics of the heavy fields, their ground state can be approximated to be supersymmetric even during inflation. Thus, we can integrate them out by using their SUSY equations of motion.

Firstly, we can set $\mathcal{V}$ to zero since it contains only heavy fields and with zero VEVs. $A_{\mu}$ in $\mathcal{V}$ cannot have non-zero VEV due to Lorentz invariance. The $D$-scalar in $\mathcal{V}$ is an order parameter for SUSY and hence $\langle D\rangle=0$ for SUSY ground state. Of course the fermions in $\mathcal{V}$ have vanishing VEVs. This leaves us with only the following terms in the action:

$$
\begin{aligned}
\mathcal{S}_{5}= & \int d^{4} x \int_{0}^{L} d x_{5}\left[\int d^{4} \theta\left\{\frac{1}{2}(\Phi+\bar{\Phi})^{2}+\overline{\mathcal{H}}^{c} \mathcal{H}^{c}+\overline{\mathcal{H}} \mathcal{H}\right\}\right. \\
& \left.+\int d^{2} \theta\left\{\mathcal{H}^{c}\left(m+\partial_{5}-\frac{g_{5}}{\sqrt{2}} \Phi\right) \mathcal{H}+\lambda(\mathcal{H}-v)^{2} \delta\left(x_{5}\right)+\lambda^{\prime}\left(\mathcal{H}-v^{\prime}\right)^{2} \delta\left(x_{5}-L\right)\right\}+\text { h.c. }\right] .
\end{aligned}
$$

The heavy charged matter fields $\mathcal{H}$ and $\mathcal{H}^{c}$, with $5 \mathrm{D}$ bulk masses $m \gtrsim m_{K K}$, can now be integrated out by imposing the following SUSY constraints:

$$
\frac{\partial W}{\partial \mathcal{H}}=0=\frac{\partial W}{\partial \mathcal{H}^{c}} .
$$

Thus, we obtain the following $4 \mathrm{D}$ effective action $^{4}$ for $\Phi$,

$$
\begin{aligned}
\mathcal{S}_{4}= & \int d^{4} x\left[\int d^{4} \theta \frac{1}{2}(\Phi+\bar{\Phi})^{2}\right. \\
& \left.+\int d^{2} \theta\left(W_{0}+\lambda \frac{v^{2} e^{-m L} e^{\frac{g L}{\sqrt{2}} \Phi}+v^{\prime 2} e^{m L} e^{-\frac{g L}{\sqrt{2}} \Phi}-2 v v^{\prime}}{e^{m L} e^{-\frac{g L}{\sqrt{2}} \Phi}+e^{-m L} e^{\frac{g L}{\sqrt{2}} \Phi}}+\text { h.c. }\right)\right],
\end{aligned}
$$

where we take $\lambda=\lambda^{\prime}$ for technical simplicity. The derivatives on $\mathcal{H}$ and $\mathcal{H}^{c}$ at the boundaries in (3.3) are evaluated by taking into account their orbifold parity (even and odd, respectively).

The Kähler potential in (3.5) displays shift symmetry for $A_{5}$, which is the imaginary part of the scalar component of $\Phi$. However, integrating out the charged hypermultiplet using (3.4) also generates shift symmetry violating terms in the Kähler potential. These corrections are however functions of $g L \Phi$ and suppressed by $e^{-m L}$, our modest expansion parameter. Thus, they contribute to the scalar potential only with $\frac{\Phi}{f}$-dependence $\left(f \equiv \frac{1}{g L}\right)$, not changing its qualitative form. Furthermore, the $e^{-m L}$ suppression makes these corrections sub-dominant and hence we neglect them here.

\footnotetext{
${ }^{3}$ As can be seen in section 4.1, $\frac{V_{\text {inf }}^{1 / 4}}{m_{K K}} \sim \frac{v L}{\sqrt{f}} e^{-m L} \sim \frac{1}{\sqrt{g}} e^{-m L} v L^{3 / 2}$ which is small due to the smallness of $e^{-m L}$ and the hypermultiplet boundary VEVs $\sim v$.

${ }^{4}$ Here, all the $4 \mathrm{D}$ fields are in canonical normalization. The $4 \mathrm{D}$ gauge coupling $g$ is defined as: $\frac{1}{g^{2}}=\frac{L}{g_{5}^{2}}$.
} 


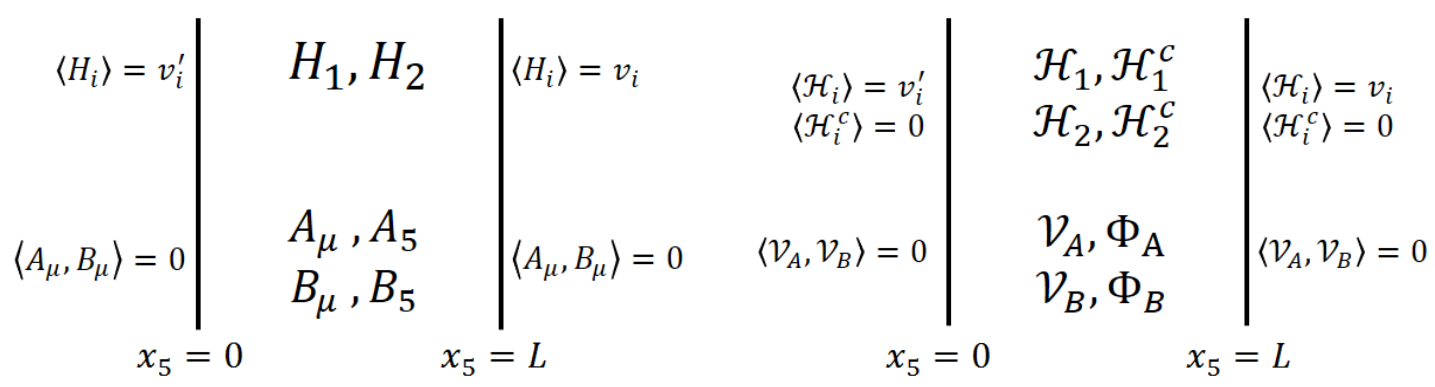

(a) Non-SUSY.

(b) SUSY.

Figure 2. Bi-axion inflation field content: (a) non-SUSY and (b) SUSY version. See text for details.

The superpotential is the source of shift symmetry breaking for $A_{5}$ which is naturally suppressed by $e^{-m L}$ for $m L \gtrsim 1$ (see e.g. for $v \sim v^{\prime}$ ). This is a generic feature of extranatural inflation scenario where the compact extra dimension effectively acts as a "filter" for any far-UV physics by suppressing its contribution by $e^{-M_{\mathrm{UV}} L} . W_{0}$ is a constant term in the superpotential which is relevant only in the presence of gravity, as we will see in section 3.3.

\subsection{Bi-axion generalization to realize $f_{\text {eff }}>M_{\mathrm{Pl}}$}

As mentioned in section 1 , in order to have $f_{\text {eff }}>M_{\mathrm{Pl}}$, we need to introduce two axions in such a way that one of their linear combinations has an effective super-Planckian field range. The non-SUSY version of bi-axion inflation has the setup as shown in figure 2a. There are two gauge fields $\left(A_{M}, B_{M}\right)$ with only $\left(A_{5}, B_{5}\right)$ having zero modes (by suitably assigning boundary conditions). The scalar fields $H_{1}$ and $H_{2}$ are charged under the gauge groups as $(0,1)$ and $(1, N)$, respectively. This field content can now be embedded into the respective 5D SUSY multiplets, as shown in figure $2 \mathrm{~b}$. By extending the construction from section 3.1, the full $5 \mathrm{D}$ action in this case can be obtained as follows:

$$
\begin{aligned}
\mathcal{S}_{5}= & \int d^{4} x \int_{0}^{L} d x_{5}\left[\int d^{2} \theta\left(\frac{1}{4} \mathcal{W}_{A, \alpha}^{2}+\frac{1}{4} \mathcal{W}_{B, \alpha}^{2}\right)+\right.\text { h.c. } \\
& +\int d^{4} \theta\left\{\partial_{5} \mathcal{V}_{A}-\frac{1}{\sqrt{2}}\left(\Phi_{A}+\bar{\Phi}_{A}\right)\right\}^{2}+\left\{\partial_{5} \mathcal{V}_{B}-\frac{1}{\sqrt{2}}\left(\Phi_{B}+\bar{\Phi}_{B}\right)\right\}^{2} \\
& +\int d^{4} \theta\left\{\left(\mathcal{H}_{1}^{c} e^{g_{5} \mathcal{V}_{B}} \overline{\mathcal{H}}_{1}^{c}+\overline{\mathcal{H}}_{1} e^{-g_{5} \mathcal{V}_{B}} \mathcal{H}_{1}\right)+\left(\mathcal{H}_{2}^{c} e^{g_{5}\left(\mathcal{V}_{A}+N \mathcal{V}_{B}\right)} \overline{\mathcal{H}}_{2}^{c}+\overline{\mathcal{H}}_{2} e^{-g_{5}\left(\mathcal{V}_{A}+N \mathcal{V}_{B}\right)} \mathcal{H}_{2}\right)\right\} \\
& +\int d^{2} \theta\left\{\mathcal{H}_{1}^{c}\left(m+\partial_{5}-\frac{g_{5}}{\sqrt{2}} \Phi_{B}\right) \mathcal{H}_{1}+\mathcal{H}_{2}^{c}\left(m+\partial_{5}-\frac{g_{5}}{\sqrt{2}}\left(\Phi_{A}+N \Phi_{B}\right)\right) \mathcal{H}_{2}\right\}+\text { h.c. } \\
& +\int d^{2} \theta\left\{\lambda_{1}\left(\mathcal{H}_{1}-v_{1}\right)^{2} \delta\left(x_{5}\right)+\lambda_{1}^{\prime}\left(\mathcal{H}_{1}-v_{1}^{\prime}\right)^{2} \delta\left(x_{5}-L\right)\right\}+\text { h.c. } \\
& \left.+\int d^{2} \theta\left\{\lambda_{2}\left(\mathcal{H}_{2}-v_{2}\right)^{2} \delta\left(x_{5}\right)+\lambda_{2}^{\prime}\left(\mathcal{H}_{2}-v_{2}^{\prime}\right)^{2} \delta\left(x_{5}-L\right)\right\}+\text { h.c. }\right] .
\end{aligned}
$$


Similarly to how (3.5) was obtained starting from (3.1) and (3.2) in the previous section, we can obtain the $4 \mathrm{D}$ effective Kähler potential and superpotential for the two axion supermultiplets $\left(\Phi_{A}, \Phi_{B}\right)$ as follows:

$$
\begin{aligned}
K= & \frac{1}{2}\left(\Phi_{A}+\bar{\Phi}_{A}\right)^{2}+\frac{1}{2}\left(\Phi_{B}+\bar{\Phi}_{B}\right)^{2} \\
W= & W_{0}+\lambda_{1} \frac{v_{1}^{2} e^{-m L} e^{\frac{g L}{\sqrt{2}}\left(\Phi_{A}+N \Phi_{B}\right)}+v_{1}^{\prime 2} e^{m L} e^{-\frac{g L}{\sqrt{2}}\left(\Phi_{A}+N \Phi_{B}\right)}-2 v_{1} v_{1}^{\prime}}{e^{m L} e^{-\frac{g L}{\sqrt{2}}\left(\Phi_{A}+N \Phi_{B}\right)}+e^{-m L} e^{\frac{g L}{\sqrt{2}}\left(\Phi_{A}+N \Phi_{B}\right)}} \\
& +\lambda_{2} \frac{v_{2}^{2} e^{-m L} e^{\frac{g L}{\sqrt{2}} \Phi_{B}}+v_{2}^{\prime 2} e^{m L} e^{-\frac{g L}{\sqrt{2}} \Phi_{B}}-2 v_{2} v_{2}^{\prime}}{e^{m L} e^{-\frac{g L}{\sqrt{2}} \Phi_{B}}+e^{-m L} e^{\frac{g L}{\sqrt{2}} \Phi_{B}}}
\end{aligned}
$$

We would like to highlight here that in (3.6), and hence also in (3.7), all the scales and field ranges are sub-Planckian.

\subsection{Adding SUGRA and identifying the SUSY vacuum}

We have not considered the effects of gravity so far in obtaining the $\mathcal{L}_{4, \text { eff }}\left(\Phi_{A}, \Phi_{B}\right)$ of (3.7). But now we can use this 4 D effective $K$ and $W$ to compute the SUGRA scalar potential ( $\left.V_{\text {SUGRA }}\right)$ directly in 4D (see appendix A). With this strategy, from effective field theory

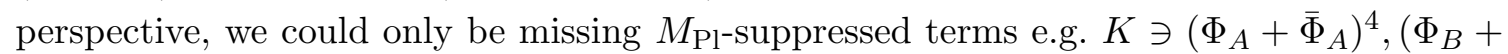
$\left.\bar{\Phi}_{B}\right)^{4}$. In the case of SUSY bi-axion model, as highlighted below (3.7), the range (and hence also the VEVs) of fields in $\Phi_{A}$ and $\Phi_{B}$ is sub-Planckian, thus making the abovementioned $M_{\mathrm{Pl}}$-suppressed terms also sub-dominant. We would like to highlight here that

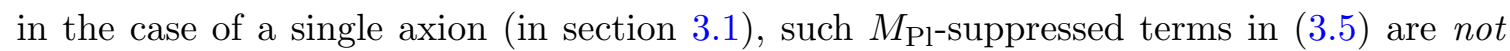
sub-dominant due to the super-Planckian range of the fields. Hence the truncation of the $\Phi / M_{\mathrm{Pl}}$ expansion is uncontrolled in this case. In section 5 , we will see that higher order Kähler interactions can have interesting observable effects if they are stronger than $M_{\mathrm{Pl}^{-} \text {-suppressed. }}$

The $W_{0}$ parameter in (3.7) is now physical, due to the presence of gravity, and it will contribute to the vacuum energy. We will consider a boundary-localized contribution to $W_{0}$ such that the net post-inflationary vacuum energy is (approximately) zero.

In order for the inflationary picture to be compatible with low energy SUSY (broken only at a scale somewhat above the EW scale) and approximately zero cosmological constant as observed today, the vacuum of post-inflationary dynamics should be SUSYpreserving and with zero vacuum energy. Thus, it seems that the inflation endpoint has to (approximately) satisfy the following three conditions: (1) unbroken SUSY $\left(\left\langle D_{\Phi_{i}} W\right\rangle=0\right)$, (2) zero vacuum energy $(\langle W\rangle=0)$, and (3) local minimum ${ }^{5}$ of $V_{\text {SUGRA }}$. However, as shown below, (1) and (2) automatically imply (3), i.e. a point in the field space satisfying $D_{\Phi_{i}} W=0$ and $W=0$ implies that it is automatically at a local minimum of $V_{\text {SUGRA }}$, so we do not bother to check (3) further.

\footnotetext{
${ }^{5}$ Global minimum can be separated enough in the field space from this local minimum such that the decay via tunneling does not happen even on the cosmological timescales.
} 
Consider, for simplicity, a single chiral superfield $\Phi$ for which $V_{\text {SUGRA }}$ is

$$
V=e^{K}\left(K_{\Phi \bar{\Phi}}^{-1}\left|D_{\Phi} W\right|^{2}-3|W|^{2}\right) .
$$

Now, for $D_{\Phi} W=0$ and $W=0$, one can clearly see that,

$$
\partial_{\Phi} V=0=\partial_{\bar{\Phi}} V, \partial_{\bar{\Phi}} \partial_{\Phi} V=e^{K} K_{\Phi \bar{\Phi}}^{-1}\left|\partial_{\Phi} D_{\Phi} W\right|^{2}, \partial_{\Phi}^{2} V=0=\partial_{\bar{\Phi}}^{2} V
$$

and for $\Phi=(\eta+i \phi) / \sqrt{2}$,

$$
\partial_{\eta} V=0=\partial_{\phi} V, \partial_{\eta}^{2} V=\partial_{\phi}^{2} V=\frac{1}{2} e^{K} K_{\Phi \bar{\Phi}}^{-1}\left|\partial_{\Phi} D_{\Phi} W\right|^{2} .
$$

Thus, for $K_{\Phi \bar{\Phi}}^{-1} \geq 0$ and $K_{\bar{\Phi} \Phi \Phi}, K_{\bar{\Phi} \Phi \bar{\Phi} \Phi}$ finite, (3.10) implies a local minimum of $V_{\text {SUGRA }}$. The same proof can be applied for multiple chiral superfields $\Phi_{i}$, with $K_{\Phi_{i} \bar{\Phi}_{i}}^{-1} \geq 0$ in the mass basis and no singularities in higher derivatives of $K$. These conditions are satisfied in our cases of interest, since we mostly have $K_{\Phi_{i} \bar{\Phi}_{i}}^{-1} \approx 1$ (see (3.7)) with corrections suppressed by high scales $\Lambda$ and small field VEVs (see section 5.1).

Furthermore, the conditions $D_{\Phi_{i}} W=0$ and $W=0$ are equivalent to the conditions

$$
\partial_{\Phi_{i}} W=0, W=0,
$$

since $D_{\Phi_{i}} W=\partial_{\Phi_{i}} W+\left(\partial_{\Phi_{i}} K\right) W$. This hugely simplifies identifying the inflation endpoint analytically. The conditions $\partial_{\Phi_{A}} W=0=\partial_{\Phi_{B}} W$ can be satisfied for the superpotential in (3.7) minimally by the following choice for the parameters ${ }^{6}$ that govern the hypermultiplet VEVs at the boundaries (see (3.6)) :

$$
v_{1}=v_{1}^{\prime}=v_{2} \equiv v, v_{2}^{\prime} \sim v e^{-m L} .
$$

In order to avoid having significant fine-tuning for choosing $v_{2}^{\prime} \sim v e^{-m L}$, we consider $e^{-m L} \sim \mathcal{O}(1)$, while still having $e^{-m L}<1$ for valid perturbative expansion (e.g. $e^{-m L} \approx$ $1 / 3$ with $m L \approx 1.1$ ). With the choice of parameters $v_{i}, v_{i}^{\prime}$ as in (3.12), and after doing a change of basis from $\left(\Phi_{A}, \Phi_{B}\right)$ to $\left(\Phi_{h}, \Phi_{l}\right)$ as defined by

$$
\Phi_{h} \equiv \Phi_{B}+\frac{1}{N} \Phi_{A}, \Phi_{l} \equiv \Phi_{A}-\frac{1}{N} \Phi_{B}
$$

the superpotential from (3.7) becomes

$$
\begin{aligned}
\frac{W}{v^{2}}= & \frac{W_{0}}{v^{2}}+\lambda_{1}\left[1-\frac{1}{\cosh \left(m L-\frac{g L}{\sqrt{2}} N \Phi_{h}\right)}\right] \\
& -2 \lambda_{2} e^{-2 m L} e^{\frac{g L}{\sqrt{2}}\left(\Phi_{h}-\frac{\Phi_{l}}{N}\right)}\left[1-\cosh \left(\frac{g L}{\sqrt{2}}\left(\Phi_{h}-\frac{\Phi_{l}}{N}\right)\right)\right]+\mathcal{O}\left(e^{-4 m L}\right) .
\end{aligned}
$$

\footnotetext{
${ }^{6}$ The simplest choice with $v_{1}=v_{1}^{\prime}=v_{2}=v_{2}^{\prime}$ does not admit a solution to $\partial_{\Phi_{A}} W=0=\partial_{\Phi_{B}} W$ when restricted to sub-Planckian field values.
} 
Now, we can identify the required Minkowski SUSY endpoint of inflation. Firstly, we identify VEVs of all the scalars, $\Phi_{k}=\frac{1}{\sqrt{2}}\left(\eta_{k}+i \phi_{k}\right)$, at inflation end by solving $\partial_{\Phi_{k}} W=0$ to obtain

$$
\left\langle\phi_{l}\right\rangle=0=\left\langle\phi_{h}\right\rangle,\left\langle\eta_{h}\right\rangle \approx \frac{f m L}{N},\left\langle\eta_{l}\right\rangle \approx f m L,
$$

with $f \equiv \frac{2}{g L}$. Then, plugging these VEVs back into (3.14), we can enforce $\langle W\rangle=0$. This self-consistently demands $W_{0}$ to be chosen to cancel the terms in (3.14) sub-dominant in $e^{-m L}$, i.e.

$$
W_{0} \sim v^{2} \cdot \mathcal{O}\left(e^{-4 m L}\right),
$$

where, as mentioned below (3.12), $e^{-m L}$ is our modest expansion parameter.

One can clearly see from (3.14) that $W \approx W\left(N \Phi_{h}, \Phi_{l} / N\right)$, for $N \gg 1$, and hence the scalar potential will be of the form

$$
V \approx V\left(\frac{N \eta_{h}}{f}, \frac{N \phi_{h}}{f}, \eta_{l}, \frac{\phi_{l}}{N f}\right)
$$

Due to the $e^{K}$ contribution from $V_{\mathrm{SUGRA}}$, and that $K \ni \frac{1}{2}\left(\Phi_{l}+\bar{\Phi}_{l}\right)^{2}$ has $\eta_{l^{-}}$but no $\phi_{l}$-dependence, the potential along $\eta_{l}$ varies over $M_{\mathrm{Pl}}$, and not $N f>M_{\mathrm{Pl}}$. As we will detail in section 4.1, from (3.17) we can power-count $m_{\eta_{h}}, m_{\phi_{h}} \sim H_{\text {inf }} \cdot \mathcal{O}\left(\frac{N^{2}}{f}\right) \gg H_{\text {inf }}$ and $m_{\eta_{l}} \sim \mathcal{O}\left(H_{\text {inf }}\right)$, while only $m_{\phi_{l}} \ll H_{\text {inf. }}$. We will show in the section 4.1 that after integrating out the heavy fields $\left(\eta_{h}, \phi_{h}, \eta_{l}\right)$, we get $V_{\text {eff }}\left(\frac{\phi_{l}}{N f}\right)$ such that $\phi_{l}$ has an effective super-Planckian field range: $f_{\text {eff }}=N f>M_{\mathrm{Pl}}$ with $f<M_{\mathrm{Pl}}$ and $N \gg 1$. Thus, we expect that the SUSY vacuum $\phi_{l}=0$ can be approached from some $\phi_{l}^{\text {initial }} \sim \mathcal{O}(N f)$ along a slow-roll potential with the slow-roll parameters $\epsilon, \eta \sim\left(\frac{M_{\mathrm{Pl}}}{N f}\right)^{2} \ll 1$.

As in the case of single axion supermultiplet (see below (3.5)), integrating out the charged hypermultiplets in (3.6) also generates shift symmetry violating terms in the Kähler potential in (3.7). Similar to the case of single axion supermultiplet, these corrections are functions of $\frac{\Phi_{l}}{f_{\mathrm{eff}}}$ which maintain the form of the effective inflationary potential, $V_{\text {eff }}\left(\frac{\phi_{l}}{f_{\mathrm{eff}}}\right)$, i.e. effective super-Planckian field range for $\phi_{l}$. Furthermore, they are suppressed by powers of $e^{-m L}$, our modest expansion parameter, which makes them sub-dominant and hence we neglect them here.

\section{Inflationary history}

In this section, we describe various aspects of the inflationary history from our SUSY biaxion model. Here we discuss the inflationary trajectory, SUSY breaking during inflation, accounting for the SUSY breaking vacuum that we occupy today, and the interplay of different fine-tunings within this model.

\subsection{Inflationary trajectory}

In order to identify the inflationary trajectory and the effective potential along it, we first consider the general problem where a potential depends on some heavy fields $\vec{H}$ and some 
light fields $\vec{L}$. We expand the potential to quadratic order in $\vec{H}$ while keeping it to all orders in $\vec{L}$ :

$$
V(\vec{H}, \vec{L})=V(\langle\vec{H}\rangle, \vec{L})+A_{i}(\vec{L}) \cdot \delta H_{i}+\frac{1}{2} m_{i j}^{2}(\vec{L}) \cdot \delta H_{i} \cdot \delta H_{j}+\mathcal{O}\left(\delta H^{3}\right) .
$$

Here, $\langle\vec{H}\rangle$ are VEVs of the heavy fields at the post-inflationary SUSY vacuum, while $\delta \vec{H}$ are the fluctuations away from $\langle\vec{H}\rangle$ in the course of inflation. The expansion coefficients are

$$
A_{i}(\vec{L}) \equiv \partial_{H_{i}} V(\langle\vec{H}\rangle, \vec{L}), m_{i j}^{2}(\vec{L}) \equiv \partial_{H_{i}} \partial_{H_{j}} V(\langle\vec{H}\rangle, \vec{L}),
$$

which are functions of $\vec{L}$. We can now integrate out the heavy fields by extremizing (4.1) with respect to the heavy fluctuations $\delta \vec{H}$, for given light fields $\vec{L}$, thereby getting an effective potential for $\vec{L}$ as

$$
V_{\mathrm{eff}}(\vec{L}) \approx V(\langle\vec{H}\rangle, \vec{L})+\frac{1}{2}\left[A_{i}\left(m^{2}\right)_{i j}^{-1} A_{j}\right](\vec{L}) .
$$

In the case of our SUSY bi-axion inflation model, the heavy and light fields along the inflationary trajectory are, respectively,

$$
\vec{H}=\left(\eta_{h}, \phi_{h}, \eta_{l}\right), \vec{L}=\left(\phi_{l}\right) .
$$

The coefficients $A_{i}$ and mass matrix $m_{i j}^{2}$ in $V_{\text {SUGRA }}$ (A.2), for the superpotential (3.14), scale parametrically as follows:

$$
\begin{aligned}
A_{i}(\vec{L}) & \sim\left(N^{2}, N^{2}, \frac{1}{N}\right) \frac{V(\langle\vec{H}\rangle, \vec{L})}{f}, \\
m_{i j}^{2}(\vec{L}) & \sim\left(\begin{array}{ccc}
N^{4} & N^{2} & N \\
N^{2} & N^{4} & 0 \\
N & 0 & f^{2}
\end{array}\right) \frac{V(\langle\vec{H}\rangle, \vec{L})}{f^{2}},
\end{aligned}
$$

where the indices $i, j$ run over $\vec{H}$ in the same order as in (4.4). We can now estimate the parametric size of the heavy fluctuations during inflation as

$$
\langle\delta \vec{H}\rangle \sim\left(\frac{f}{N^{2}}, \frac{f}{N^{2}}, \frac{1}{N f}\right) .
$$

This then implies that the $\mathcal{O}\left(\delta H^{3}\right)$ term that we dropped in (4.1) is sub-dominant, suppressed by the small parameters $\frac{1}{N}$ and $\frac{1}{N f}$, and hence can be ignored along the inflationary trajectory.

The mass eigenvalues of the heavy fluctuations $\delta \vec{H}$ are as follows:

$$
\begin{aligned}
& m_{1,2}^{2} \approx m_{\eta_{h}, \phi_{h}}^{2} \sim N^{4} \frac{V(\langle\vec{H}\rangle,\langle\vec{L}\rangle)}{f^{2}} \sim \frac{N^{4}}{f^{2}} H_{\mathrm{inf}}^{2}, \\
& m_{3}^{2} \approx m_{\eta_{l}}^{2} \sim V(\langle\vec{H}\rangle, \vec{L}) \sim H_{\mathrm{inf}}^{2}(\vec{L}) .
\end{aligned}
$$


Thus, the heavy mass-squared eigenvalues are all positive and much larger than $m_{\phi_{l}}^{2} \sim$ $\frac{V(\langle\vec{H}\rangle \vec{L})}{N^{2} f^{2}}$. Hence, we can integrate out the heavy fluctuations $\delta \vec{H}$ all along the inflationary trajectory yielding

$$
V_{\mathrm{eff}}\left(\phi_{l}\right) \approx V\left(\langle\vec{H}\rangle, \frac{\phi_{l}}{N f}\right) \cdot\left[1+\mathcal{O}(1)+\mathcal{O}\left(\frac{1}{N^{2} f^{2}}\right)\right] .
$$

Here, the second term comes from integrating out the mass eigenstates $\left(H_{1}, H_{2}\right) \approx\left(\eta_{h}, \phi_{h}\right)$ while the third term comes from integrating out $H_{3} \approx \eta_{l}$. Since the contribution from $\left(H_{1}, H_{2}\right)$ is of the same order as $V\left(\langle\vec{H}\rangle, \frac{\phi_{l}}{N f}\right)$, we perform the integration out of $\delta \vec{H}$ numerically. Firstly, we verify that as suggested by the parametric estimates in (4.5) and (4.7), the heavy mass-squared eigenvalues are indeed much larger than $m_{\phi_{l}}^{2}$ all along the inflationary trajectory. The numerically computed $V_{\text {eff }}\left(\phi_{l}\right)$ is as shown in figure 3 . It has the following approximate analytic form,

$$
V_{\text {eff }}\left(\phi_{l}\right) \sim H_{\text {inf }}^{2}\left(1-\cos \frac{\phi_{l}}{N f}\right), H_{\text {inf }} \sim \lambda_{2} \frac{v^{2}}{f} e^{-2 m L},
$$

which is the leading contribution in terms of the small parameters $1 / N, f$ and $e^{-m L}$. Thus, the SUSY bi-axion model effectively provides an approximate Natural Inflation model with $f_{\text {eff }}=N f>M_{\mathrm{Pl}}$, where the best-fit values are [49]

$$
f_{\mathrm{eff}}=N f \sim 10 M_{\mathrm{Pl}}, V_{\mathrm{inf}}^{1 / 4} \sim 10^{16} \mathrm{GeV} .
$$

The precise inflationary potential, $V_{\text {eff }}\left(\phi_{l}\right)$, does contain "higher harmonics" in $\frac{\phi_{l}}{N f}$. Although, these do not affect the qualitative features as outlined above, they can play an important role in precision fitting to the CMB data which we will explore in a future work.

As can be seen from (4.7), the heavy fields $\eta_{h}, \phi_{h}$ are much heavier than $H_{\text {inf }}$ :

$$
m_{\eta_{h}}, m_{\phi_{h}} \sim H_{\text {inf }} \frac{N^{2}}{f} .
$$

While sinflaton $\left(\eta_{l}\right)$, the real scalar partner of the inflaton, has an intermediate mass,

$$
m_{\eta_{l}} \sim \mathcal{O}\left(H_{\mathrm{inf}}\right)
$$

about which we will discuss more in section 5.1.1.

\subsection{SUSY breaking during inflation}

As mentioned earlier in section 2, the approximate de Sitter geometry during inflation requires it to be an excited state with spontaneous SUSY on top of the post-inflationary SUSY vacuum. The SUSY order parameters (see appendix A) for $\Phi_{h}$ and $\Phi_{l}$ evaluated along the inflationary trajectory are

$$
\begin{aligned}
&\left.D_{\Phi_{h}} W\right|_{\left\langle\Phi_{h}\right\rangle,\left\langle\eta_{l}\right\rangle} \sim \lambda_{2} \frac{v^{2}}{f} e^{-2 m L} e^{-i \frac{\phi_{l}}{N f}}\left(1-\cos \frac{\phi_{l}}{N f}\right), \\
&\left.\left.D_{\Phi_{l}} W\right|_{\left\langle\Phi_{h}\right\rangle,\left\langle\eta_{l}\right\rangle} \sim \mathcal{O}\left(\frac{1}{N}\right) \cdot D_{\Phi_{h}} W\right|_{\left\langle\Phi_{h}\right\rangle,\left\langle\eta_{l}\right\rangle} .
\end{aligned}
$$




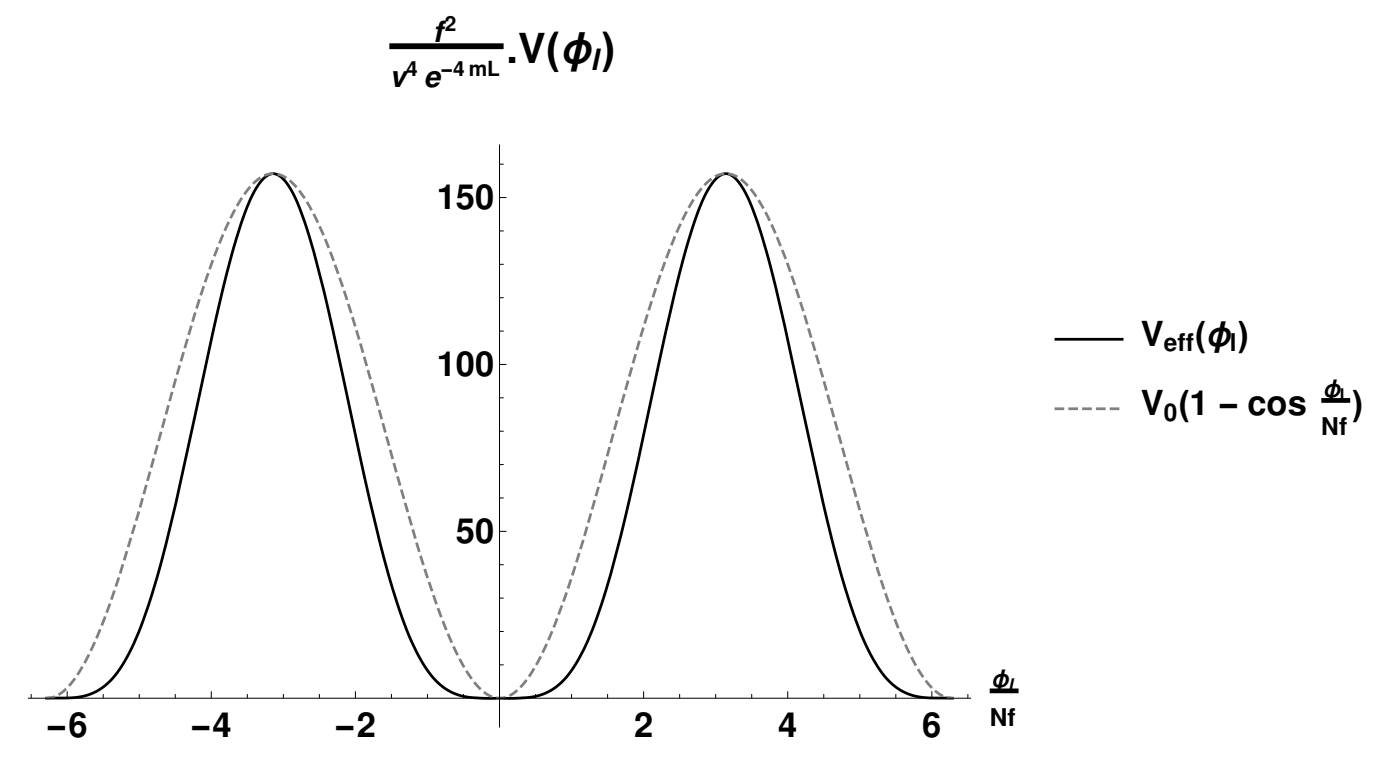

Figure 3. The dark line refers to the effective inflationary potential $V_{\text {eff }}\left(\phi_{l}\right)$ after numerically integrating out the heavy fields $\eta_{h}, \phi_{h}, \eta_{l}$ all along the inflationary trajectory. For comparison, a pure cosine potential with the magnitude matching to that of $V_{\text {eff }}\left(\phi_{l}\right)$ is plotted as the dashed line. Inflation can start close to the hilltop of $V_{\mathrm{eff}}\left(\phi_{l}\right)$, with $V_{\mathrm{inf}} \sim \frac{v^{4} e^{-4 m L}}{f^{2}}$.

Here, we can clearly see that the SUSY order parameters are zero only at the vacuum (i.e. $\left.\phi_{l}=0\right)$.

As described in appendix A, the massless Goldstino of spontaneous SUSY, which is "eaten" by gravitino to become massive, is given by the linear combination of all the fermions weighted by the respective SUSY order parameters: $\psi_{\text {Goldstino }} \propto\left\langle D_{\Phi_{i}} W\right\rangle \cdot \psi_{i}$. Hence, the SUSY order parameters during inflation (4.13) imply that,

$$
\psi_{\text {Goldstino }} \sim \psi_{h}+\mathcal{O}\left(\frac{1}{N}\right) \cdot \psi_{l},
$$

i.e. the Goldstino during inflation belongs mostly to the heavy sector $\left(\Phi_{h}\right)$ and not to the inflaton sector $\left(\Phi_{l}\right)$. In other words, SUSY during inflation is caused mostly by the heavy sector $\left(\Phi_{h}\right)$. This feature of our SUSY bi-axion model is very similar to the KLR model discussed in section 2 where the Goldstino during inflation belongs solely to the heavy sector $(S)$ (see $(2.4))$.

\subsection{SUSY breaking after inflation}

We need to account for the post-inflationary SUSY vacuum that we occupy today which we have neglected so far. In this section, we look for any possibly significant effects of this SUSY vacuum on the inflationary dynamics. We consider a boundary-localized Polonyitype sector with

$$
\delta K_{4}=\bar{X} X-\frac{(\bar{X} X)^{2}}{\Lambda^{2}}, \delta W_{4}=\Lambda_{\text {SUSY }}^{2} X
$$

which undergoes spontaneous SUSY at a scale $\sim \Lambda_{\text {SUSY }}$. Consider the SUSY from this hidden sector to be minimally communicated to the Standard Model via $M_{\mathrm{Pl}}$ suppressed 
interactions which then implies an intermediate scale SUSY $\Lambda_{\text {SUSY }} \sim \sqrt{v_{\text {weak }} \cdot M_{\mathrm{Pl}}} \sim$ $10^{11} \mathrm{GeV}$. We expect that as long as $\Lambda_{\text {SUSY }} \ll H_{\text {inf }}$ or even $V_{\text {inf }}^{1 / 4}$, the effect of this SUSY $X$-sector on the inflationary dynamics will be negligible. (See also e.g. [60].) We show below that indeed this expectation is borne out here.

The scalar field $X$ in the Polonyi sector gets $\mathcal{O}\left(H_{\text {inf }}\right)$ mass during inflation, similar to that of $\eta_{l}$. Thus, $X$ can be added to the "heavy" fields $\vec{H}$ of (4.4) and essentially the same procedure as described in section 4.1 can be repeated to integrate them out along the inflationary trajectory. The tadpole and mass matrix terms (see (4.2)) involving $X$ field scale as follows:

$$
\begin{aligned}
A_{X}\left(\phi_{l}\right) & \sim \Lambda_{\text {SUSY }}^{2} H_{\mathrm{inf}}\left(\phi_{l}\right) \cdot f, & m_{X X}^{2}\left(\phi_{l}\right) & \sim H_{\mathrm{inf}}^{2}\left(\phi_{l}\right), \\
m_{\eta_{h} X}^{2}\left(\phi_{l}\right) & \sim m_{\phi_{h} X}^{2}\left(\phi_{l}\right) \sim \Lambda_{\text {SUSY }}^{2} H_{\mathrm{inf}}\left(\phi_{l}\right) \cdot N, & m_{\eta_{l} X}^{2}\left(\phi_{l}\right) & \sim \Lambda_{\text {SUSY }}^{2} H_{\mathrm{inf}}\left(\phi_{l}\right) \frac{1}{N} .
\end{aligned}
$$

The fluctuations of $X$ during inflation can be estimated from (4.16) as

$$
\delta X \sim \frac{\Lambda_{\text {SUSY }}^{2} f}{V_{\mathrm{inf}}^{1 / 2}\left(\phi_{l}\right)} .
$$

The $\mathcal{O}\left(\delta H^{3}\right)$ terms in (4.1) involving $X$ are hence sub-dominant, suppressed by $\frac{\Lambda_{\text {SUSY }}}{V_{\mathrm{inf}}^{1 / 4}}$. We can now integrate out $X$ following (4.3) yielding

$$
\delta V_{\text {eff }}\left(\phi_{l}\right) \sim \Lambda_{\text {SUSY }}^{4} \cdot f^{2} \cdot\left[1+\frac{1}{N^{2}} \frac{\Lambda_{\text {SUSY }}^{4}}{V_{\text {eff }}\left(\phi_{l}\right)}\right] .
$$

This $\delta V_{\text {eff }}\left(\phi_{l}\right)$ is much smaller than the $V_{\text {eff }}\left(\phi_{l}\right)$ of (4.8). Thus, as expected, for $\Lambda_{\text {SUSY }} \sim$ $10^{11} \mathrm{GeV}$ and $V_{\text {inf }}^{1 / 4} \sim 10^{16} \mathrm{GeV}$ (see (4.10)) satisfying $\Lambda_{\text {SUSY }} \ll V_{\text {inf }}^{1 / 4}$, the SUSY $X$-sector gives a negligible contribution to $V_{\text {eff }}\left(\phi_{l}\right)$ and hence does not significantly affect the inflationary dynamics.

\subsection{Interplay of electroweak, cosmological constant and superpotential tunings}

In order to have (almost) vanishing vacuum energy after the end of inflation, as discussed in section 3.3, we need to have $\langle W\rangle=0$ which can be achieved by tuning the $W_{0}$ parameter in (3.7). We also need to account for the SUSY breaking vacuum that we occupy today. Here, we evaluate this combined fine-tuning which displays an interesting interplay with the electroweak (EW) and cosmological constant (CC) fine-tunings.

Consider the SUSY hidden sector of section 4.3 which minimally communicates to the Standard Model via gravity mediation (i.e. $M_{\mathrm{Pl}}$ suppressed interactions). In order for it to address the electroweak hierarchy problem, this requires that $\frac{V_{\text {SUSY }}^{\text {today }}}{M_{\mathrm{Pl}}^{2}} \sim v_{\text {weak }}^{2} \cdot{ }^{7}$ This implies the following fine-tuning in the EW sector,

$$
T_{\mathrm{EW}} \sim \frac{v_{\mathrm{weak}}^{2} M_{\mathrm{Pl}}^{2}}{V_{\text {SUSY }}^{\text {today }}}
$$

\footnotetext{
${ }^{7} V_{\text {SUSY }}^{\text {today }}=\Lambda_{\text {SUSY }}^{4}$.
} 
which can be minimized with $\left(V_{\text {SUSY }}^{\text {today }}\right)^{1 / 4} \sim \sqrt{v_{\text {weak }} M_{\mathrm{Pl}}}$, as is standard. This SUSY sector and also $\Delta W_{0} \neq 0$ in (3.7) give contributions to the CC today as below:

$$
\mathrm{CC}=-3 \frac{\Delta W_{0}^{2}}{M_{\mathrm{Pl}}^{2}}+V_{\mathrm{SUSY}}^{\text {today }} \stackrel{(\text { obs. })}{\sim} \mathrm{meV}^{4}
$$

The two terms in the above equation have typical sizes of $\sim \frac{v^{4}}{M_{\mathrm{Pl}}^{2}}($ see $(3.16))$ and $\sim$ $v_{\text {weak }}^{2} M_{\mathrm{Pl}}^{2}$, respectively, which consist of a priori different and unrelated scales. This implies that multiple contributions to $\Delta W_{0}$, each of magnitude $\sim \frac{v^{2}}{M_{\mathrm{Pl}}}$, must first cancel to within $\sqrt{V_{\text {SUSY }}^{\text {today }}}$. Hence, we have the following fine-tuning in the contributions to $W_{0}$ :

$$
T_{W_{0}} \sim \frac{\sqrt{V_{\text {SUSY }}^{\text {today }}} M_{\mathrm{Pl}}}{v^{2}} .
$$

Once the two terms on the right hand side of (4.20) are of the same order, they still have to cancel to give $\mathrm{CC} \sim \mathrm{meV}^{4}$ as observed today. This amounts to having the following usual CC fine-tuning:

$$
T_{\mathrm{CC}} \sim \frac{\mathrm{meV}^{4}}{V_{\text {SUSY }}^{\text {today }}}
$$

As can be seen from (4.19) and (4.22), the EW and CC fine-tunings favor SUSY at low-scale. However, (4.21) shows that the $W_{0}$ fine-tuning displays preference for SUSY at high-scale! But, the net fine-tuning, assuming that these three are independent of each other, is

$$
T_{\text {net }}=T_{\mathrm{EW}} \times T_{W_{0}} \times T_{\mathrm{CC}} \sim \frac{v_{\text {weak }}^{2} M_{\mathrm{Pl}}^{3} \mathrm{meV}^{4}}{v^{2}}\left(V_{\text {SUSY }}^{\text {today }}\right)^{-3 / 2} .
$$

This shows a net preference for SUSY at low-scale, namely close to the EW scale.

Our considerations here are reminiscent of comparable tuning issues that arise in highscale string-derived SUGRA theories, in particular the necessary existence of a high-scale $W_{0}$ which makes the tuning worse. See [61] for a review. For a sample choice of the parameters, $V_{\text {SUSY }}^{\text {today }} \sim v_{\text {weak }}^{2} M_{\mathrm{Pl}}^{2}$ and $v^{2} \sim\left(0.1 M_{\mathrm{Pl}}\right)^{3}$, we see that the net tuning in (4.23) is considerable $\left(T_{\text {net }} \sim 10^{-100}\right)$, predominantly because of the Cosmological Constant Problem. However, such a residual tuning is still acceptable in the context of the anthropic principle or some as yet unknown mechanism solving this problem. See $[62,63]$ for a review.

\section{Observable signals}

In this section we discuss the phenomenology of our SUSY bi-axion model. The observable signals from this model can come in the form of primordial non-Gaussianities mediated by heavy particles, sinflaton being the prime candidate for this. Also, "higher harmonic" terms in the inflaton potential can give rise to periodic modulations in the CMB. 


\subsection{Primordial non-Gaussianities}

As first introduced in [29] and further illustrated in [30-39], a particle $X$ can mediate primordial non-Gaussianities of observable size if it (1) has $m_{X} \sim \mathcal{O}\left(H_{\text {inf }}\right)$, (2) has sufficiently strong $X(\partial \phi)(\partial \phi)$ couplings, and (3) can give tree-level contribution to inflaton 3 -point function which can come only from bosons.

\subsubsection{Sinflaton}

In the SUSY bi-axion model, mass of the sinflaton $\left(\eta_{l}\right)$ during inflation is

$$
m_{\eta_{l}} \approx \sqrt{6} H_{\mathrm{inf}}
$$

which can be seen schematically from $V_{\mathrm{SUGRA}}$ as follows:

$$
\begin{aligned}
V_{\text {SUGRA }} & =e^{K}\left(\left|D_{\Phi_{A}} W\right|^{2}+\left|D_{\Phi_{B}} W\right|^{2}-3|W|^{2}\right), \\
V\left(\eta_{l}\right) & \approx e^{\eta_{l}^{2}} V_{\mathrm{inf}} \ni \eta_{l}^{2} V_{\mathrm{inf}} \approx 3 H_{\mathrm{inf}}^{2} \eta_{l}^{2} .
\end{aligned}
$$

This contribution to $m_{\eta_{l}}$ comes from the coupling of $\eta_{l}$ to the SUSY curvature during inflation which also shows up in the KLR model as described in section 2 (see (2.5)). However, as in the case of the KLR model (see (2.7)), $m_{\eta_{l}} \sim \mathcal{O}\left(H_{\text {inf }}\right)$ is not guaranteed in our SUSY bi-axion model too. A higher order term in Kähler potential of the form

$$
K_{5} \ni \delta\left(x_{5}\right) \frac{c_{2}}{\Lambda_{2}^{2}}\left(\Phi_{A}+\bar{\Phi}_{A}\right)^{2}\left(\Phi_{B}+\bar{\Phi}_{B}\right)^{2}
$$

can give a contribution to the sinflaton mass as

$$
m_{\eta_{l}}^{2} \approx \frac{2 V_{\mathrm{inf}}}{M_{\mathrm{Pl}}^{2}}+\frac{c_{2} V_{\mathrm{inf}}}{\Lambda_{2}^{2}}=6 H_{\mathrm{inf}}^{2}\left(1+\frac{c_{2} M_{\mathrm{Pl}}^{2}}{2 \Lambda_{2}^{2}}\right) .
$$

Thus, for $\frac{c_{2}}{\Lambda_{2}^{2}} \gg \frac{1}{M_{\mathrm{Pl}}^{2}}, m_{\eta_{l}} \gg H_{\text {inf }}$ is possible. ${ }^{8}$ The effective higher order coupling (5.3) between $\Phi_{A}$ and $\Phi_{B}$ can arise radiatively via the loops of hypermultiplet $\left(H_{2}, H_{2}^{c}\right)$ which is charged under both the gauge groups as $(1, N)$. Naive dimensional analysis suggests that this loop contribution to (5.3) is

$$
\left(\frac{c_{2}}{\Lambda_{2}^{2}}\right)_{\text {loop }} \sim \frac{g^{2} N^{2}}{16 \pi^{2}} \frac{1}{m_{K K}^{2}} .
$$

Considering $N \sim \mathcal{O}(100)$ and $m_{K K} \sim M_{5} \sim 0.1 M_{\mathrm{Pl}},{ }^{9}$ we can have $\left(\frac{c_{2}}{\Lambda_{2}^{2}}\right)_{\text {loop }} \lesssim \frac{1}{M_{\mathrm{Pl}}^{2}}$ with $g \lesssim 0.1$. Thus, with $g \lesssim 0.1$, the contribution from loop-induced term (5.3) to sinflaton mass is small, thus keeping $m_{\eta_{l}} \sim \mathcal{O}\left(H_{\text {inf }}\right)$, which is crucial to get observable NG mediated by it.

\footnotetext{
${ }^{8}$ One might worry that the sub-leading term (5.3) can have a dominant effect on the sinflaton mass and whether this signals breakdown of the EFT expansion. This is however not true. The sinflaton mass in (5.1)

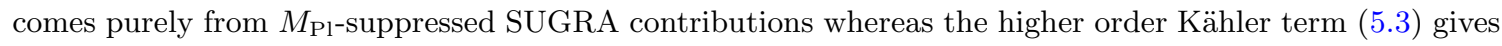
a direct coupling with suppression scale $\left(\Lambda_{2}\right)$ which can be below $M_{\mathrm{Pl}}$.

${ }^{9} M_{5} \sim M_{\mathrm{Pl}}\left(\frac{1}{M_{\mathrm{Pl}} L}\right)^{1 / 3}$ is the scale at which gravity becomes strong in $5 \mathrm{D}$.
} 
The references $[64,65]$ and [66] construct SUSY EFT of inflation with a minimal field content which does not include any scalar other than the inflaton, especially the sinflaton. This can be interpreted by the UV-completion of these EFTs having sinflaton with mass much greater than $H_{\text {inf }}$. In our SUSY bi-axion model, as can be seen from (5.4), there exists a region of parameter space where $m_{\eta_{l}} \gg H_{\text {inf }}$, which is consistent with the results of [64-66]. This parameter space corresponds to $\Lambda_{2} \ll M_{\mathrm{Pl}}$ in (5.4) or $g>0.1$ in (5.5). This feature of having a region of parameter space allowing $m_{\text {sinflaton }} \gg H_{\text {inf }}$ is also present in the KLR model described in section 2 (see (2.7)). However, in this case, the size of primordial NG suffers a severe exponential "Boltzmann-suppression" $\left(\sim e^{-\pi m_{\eta_{l}} / H_{\text {inf }}}\right)$. Below, we focus on the region where $m_{\eta_{l}} \sim \mathcal{O}\left(H_{\text {inf }}\right)$ which allows the sinflaton to be observable via primordial NG.

Even in the presence of $m_{\eta_{l}} \sim \mathcal{O}\left(H_{\text {inf }}\right), \eta_{l}$ still needs to have sufficiently strong coupling with the inflaton to mediate NG of observable size. The $V_{\text {SUGRA }}$ from (3.7) has the following coupling which violates the shift symmetry for $\phi_{l}$ and hence is very small:

$$
V_{\text {SUGRA }} \ni 10^{-3} \frac{H_{\text {inf }}}{M_{\mathrm{Pl}}} H_{\text {inf }} \eta_{l} \phi_{l}^{2} .
$$

This coupling gives rise to the primordial NG of the following typical size [37, 39]:

$$
f_{\mathrm{NL}} \sim 10^{-2} \frac{H_{\mathrm{inf}}}{M_{\mathrm{Pl}}} \lesssim 10^{-6}
$$

This is much less than the sensitivity of the proposed experiments involving 21-cm cosmology, $f_{\mathrm{NL}} \sim 10^{-2}[67,68]$, or even from more futuristic surveys, $f_{\mathrm{NL}} \sim 10^{-4}$ [69].

However, the following shift symmetry preserving, higher order, boundary-localized term in the Kähler potential,

$$
K_{5} \ni \delta\left(x_{5}\right) \frac{c_{1}}{\Lambda_{1}^{2}}\left(\Phi_{A}+\bar{\Phi}_{A}\right)^{4}
$$

can generate the following derivative coupling of sinflaton with inflaton:

$$
\mathcal{L}_{4} \ni \frac{c_{1}}{\Lambda_{1}^{2}} \eta_{l}^{2}\left(\partial \phi_{l}\right)^{2}
$$

The above coupling can give primordial NG of the size [37, 39]

$$
f_{\mathrm{NL}} \approx 0.03 c_{1}^{2} \epsilon\left(\frac{M_{\mathrm{Pl}}}{\Lambda_{1}}\right)^{4}\left(\frac{\left\langle\eta_{l}\right\rangle_{\mathrm{inf}}}{M_{\mathrm{Pl}}}\right)^{2} \lesssim 10^{-6}\left(\frac{M_{\mathrm{Pl}}}{\Lambda_{1}}\right)^{4},
$$

where the VEV of sinflaton during inflation is $\left\langle\eta_{l}\right\rangle_{\text {inf }} \approx \frac{M_{\mathrm{Pl}}^{2}}{N f} \approx 0.1 M_{\mathrm{Pl}}$, as can be calculated from $V_{\text {SUGRA }}$ using (3.7). $\epsilon$ in the above expression is the slow roll parameter of inflation which is constrained to be $\lesssim 10^{-2}$ [49]. The suppression scale $\Lambda_{1}$ in (5.8), which would be the cutoff scale on the boundaries, has to be less than $M_{5}$. Considering $M_{5} \sim \mathcal{O}(0.1) M_{\mathrm{Pl}}$, this implies that even for $\Lambda_{1}$ being very close to $M_{5}$, we can get $f_{\mathrm{NL}} \sim \mathcal{O}\left(10^{-2}\right)$. This signal can be observed at the proposed 21-cm experiments as described after (5.7). Furthermore, $\Lambda_{1}$ can be as low as the inflationary energy scale $V_{\text {inf }}^{1 / 4} \lesssim 10^{-2} M_{\mathrm{Pl}}$, while maintaining EFT control, in which case $f_{\mathrm{NL}} \sim \mathcal{O}(1)$ or even higher is also possible. 


\subsubsection{Boundary-localized gauge singlets}

It is also possible to see boundary-localized fields via primordial NG. Consider, for example, a chiral superfield $X$ localized at one of the boundaries and singlet under both the gauge groups $A$ and $B$. If it has the following Kähler potential, i.e. a direct coupling with $\Phi_{A}$ preserving its shift symmetry,

$$
K_{5} \ni \delta\left(x_{5}\right)\left[\frac{c_{X}}{\Lambda_{X}}\left(\Phi_{A}+\bar{\Phi}_{A}\right)^{2}(X+\bar{X})+\bar{X} X\right],
$$

then it has the following derivative interaction between the real scalar part of $X\left(\eta_{X}\right)$ and the inflaton:

$$
\mathcal{L}_{4} \ni \frac{c_{X}}{\Lambda_{X}} \eta_{X}\left(\partial \phi_{l}\right)^{2} .
$$

Also, analogous to the case of sinflaton as in (5.2), mass of this gauge singlet during inflation is

$$
m_{X} \approx \sqrt{3} H_{\mathrm{inf}} .
$$

(5.12) and (5.13) imply that the size of primordial NG mediated by $\eta_{X}$ is as follows [37, 39]:

$$
f_{\mathrm{NL}} \approx 0.75 c_{X}^{2} \epsilon\left(\frac{M_{\mathrm{Pl}}}{\Lambda_{X}}\right)^{2} \lesssim 10^{-2}\left(\frac{M_{\mathrm{Pl}}}{\Lambda_{X}}\right)^{2}
$$

Similar to the case of $\Lambda_{1}$ as discussed below (5.10), $\Lambda_{X} \lesssim M_{5} \sim 0.1 M_{\mathrm{Pl}}$ which can give $f_{\mathrm{NL}} \gtrsim \mathcal{O}(1)$.

If the direct coupling in (5.11) is of the form $K_{5} \ni \delta\left(x_{5}\right) \frac{c_{X}^{\prime}}{\Lambda_{X}^{2}}(\Phi+\bar{\Phi})^{2} \bar{X} X$, then it gives the interaction $\mathcal{L}_{4} \ni \frac{c_{X}^{\prime}}{\Lambda_{X}^{2}}|X|^{2}\left(\partial \phi_{l}\right)^{2}$. In this case, the $f_{\mathrm{NL}}$ mediated by $X$ has an additional suppression factor due to its VEV during inflation: $f_{\mathrm{NL}} \sim c_{X}^{2} \epsilon\left(\frac{M_{\mathrm{Pl}}}{\Lambda_{X}}\right)^{2}\left(\frac{\langle X\rangle}{\Lambda_{X}}\right)^{2}$. Hence, in order to get $f_{\mathrm{NL}}$ of an observable size, we need to have $\langle X\rangle$ during inflation to be sufficiently large as compared to $\Lambda_{X}$.

\subsection{Periodic modulations in the CMB}

Extra-dimensional realization of Natural Inflation gives the leading slowly varying inflaton potential with super-Planckian field range $\left(\sim f_{\text {eff }}=N f>M_{\mathrm{Pl}}\right)$, while also generically giving sub-leading "higher harmonic" terms oscillating over a much shorter range $\left(\sim f, f / N \ll M_{\mathrm{Pl}}\right)$. Although these higher harmonics in $V\left(\phi_{\text {inf }}\right)$ are suppressed by factors of $e^{-M L}$, they can still give observable effects in the form of primordial features with periodic modulations in the CMB power spectrum. These features, being motivated from various theoretical constructions, have been searched for in the Planck CMB data [13, 40-48].

In our SUSY bi-axion model, there exist such higher harmonics in $V\left(\phi_{l}\right)$ arising from within the model, but they are small. These can come from the sub-dominant terms in the superpotential (3.14), $\delta W=\delta W\left(e^{-m L} \cdot e^{\frac{g L}{\sqrt{2} N} \Phi_{l}}\right)$, suppressed by powers of $e^{-m L}$. This gives corrections to the inflaton potential of the form

$$
\frac{\delta V}{V_{\mathrm{inf}}} \sim e^{-2 n m L} \cos \left(n \frac{\phi_{l}}{N f}\right) .
$$


However, contributions to the periodic modulations in the CMB come only from the harmonics with $n \gg 1$ i.e. $n \sim \mathcal{O}(N)$. But, such $\cos \left(\mathcal{O}(N) \cdot \frac{\phi_{l}}{N f}\right)$ terms in the potential are hugely suppressed by $\sim e^{-2 m L \cdot \mathcal{O}(N)}$. Thus, the higher harmonics from within the SUSY bi-axion model cannot give rise to observable CMB periodic modulations.

But, let us now consider contribution from a generic heavy hypermultiplet beyond our minimal model $\left(\mathcal{H}_{3}, \mathcal{H}_{3}^{c}\right)$, with mass $M$ and charges $\left(n_{A}, n_{B}\right)$ under the gauge groups $A$ and $B$. This will give an additional term in the superpotential (3.14) as

$$
\delta W \approx 2 v^{2} e^{-M L}\left[1-e^{\frac{g L}{\sqrt{2}}\left(n_{A} \Phi_{A}+n_{B} \Phi_{B}\right)}\right],
$$

where we have taken the parameters governing boundary VEVs of $H_{3}, H_{3}^{c}$ to be equal: $v_{3}=v_{3}^{\prime}=v$. As expected, this is suppressed by $e^{-M L}$ which is "filtered" out by the extra dimension for $M \gg 1 / L$. However, as discussed below, the precision CMB observables can be sensitive to the contributions to periodic modulations sourced by such a hypermultiplet if it is not too heavy. The contribution to the inflaton potential from (5.16) is

$$
\frac{\delta V}{V_{\mathrm{inf}}} \approx n_{B} e^{2 m L} e^{-M L} \cos \left[\left(N n_{B}-n_{A}\right) \frac{\phi_{l}}{N f}\right] .
$$

The observational constraint on the size of CMB periodic modulations is $\left|\frac{\delta V}{V_{\text {inf }}}\right| \lesssim 10^{-5}$, also depending upon the higher harmonic frequency [47]. Considering $n_{B} \sim \mathcal{O}(N) \sim 100$ and $e^{-m L} \sim 1 / 3$, we can get $\left|\frac{\delta V}{V_{\text {inf }}}\right| \sim 10^{-5}$ from $M \sim 20 \times \frac{1}{L}$. This shows sensitivity of CMB periodic modulations to the charged matter much heavier than the KK scale!

The $5 \mathrm{D}$ gauge theory being non-renormalizable has a cutoff which is given by $\Lambda_{5 \mathrm{D}} \sim$ $\frac{c}{g^{2}} \frac{1}{L}$. As discussed below (5.5), we require $g \lesssim 0.1$ in order to have $m_{\eta_{l}} \sim \mathcal{O}\left(H_{\text {inf }}\right)$ for getting observable primordial NG mediated by sinflaton. Hence, for $g \lesssim 0.1$ and $c \sim \mathcal{O}(1)$, the cutoff is $\Lambda_{5 \mathrm{D}} \gtrsim 100 \times \frac{1}{L}$. Thus, charged matter beyond the minimal model with

$$
M \lesssim \frac{1}{5} \Lambda_{5 \mathrm{D}}
$$

can generate observable periodic modulations in the CMB power spectrum with $\left|\frac{\delta V}{V_{\text {inf }}}\right| \sim$ $10^{-5}$. Of course, some such heavy states are expected near the cutoff of 5D gauge theory as part of a UV-completion of our non-renormalizable effective field theory.

\section{Conclusions}

In the present work, we demonstrated the compatibility of low-energy SUSY (i.e. SUSY broken only at somewhat above the EW scale) with high-scale axionic inflation where the axionic nature of inflaton is derived from extra-dimensional gauge symmetry. The inflaton potential, in the presence of SUSY, can be generated at tree-level by charged matter in the $5 \mathrm{D}$ bulk with gauge symmetry breaking at the $5 \mathrm{D}$ boundaries. We also required that this robust gauge-theoretic origin for the inflaton satisfy the Weak Gravity Conjecture quantum gravity constraints, which are especially tight given the super-Planckian inflaton field range 
required by the data (Lyth bound). But we showed that this can be achieved by introducing two axion supermultiplets, containing a light inflaton direction having $f_{\mathrm{eff}}>M_{\mathrm{Pl}}$. The heavy sector, apart from stabilizing the inflationary trajectory, also contributes dominantly to SUSY breaking (SUSY) during inflation. The Goldstino of spontaneous SUSY during inflation lies mostly in this heavy sector.

Our SUSY bi-axion model displays an interesting interplay of electroweak (EW), cosmological constant $(\mathrm{CC})$ and superpotential $\left(W_{0}\right)$ fine-tunings after considering the SUSY vacuum we occupy today. The fine-tuning for EW and CC, as usual, prefer low-scale SUSY. The $W_{0}$ fine-tuning, however, shows preference for high-scale SUSY. We showed that the net fine-tuning is dominated by EW and CC fine-tunings and hence prefers low-scale SUSY i.e. somewhat above the EW scale.

The observable signals in our model can come in the form of primordial non-Gaussianities (NG) and periodic modulations in the CMB. The sinflaton can naturally have $\mathcal{O}\left(H_{\text {inf }}\right)$ mass via its coupling to the SUSY curvature during inflation. It can also naturally have sufficiently strong couplings with inflaton such that it can be seen via primordial NG in future $21-\mathrm{cm}$ experiments, with the measure of NG, $f_{\mathrm{NL}}$, being $\gtrsim 10^{-2}$. The sinflaton mass can receive large contributions from higher order Kähler terms which, however, can be kept sub-dominant with small enough gauge coupling. Similarly, a boundary-localized gauge singlet can have $\mathcal{O}\left(H_{\text {inf }}\right)$ mass during inflation and strong enough coupling with inflaton, via higher order Kähler couplings, thus allowing it to mediate large primordial NG with even $f_{\mathrm{NL}} \gtrsim \mathcal{O}(1)$.

Although the extra dimension acts as a "filter" for the unknown UV-completion of our non-renormalizable model, with $e^{-M L}$ suppression, the precision observables in the CMB can still probe modulating features imprinted by such heavy physics. We showed that charged matter, not far below the effective field theory cutoff of our model, can generate modulations in the inflationary potential, $\left|\frac{\delta V}{V_{\text {inf }}}\right| \sim 10^{-5}$, which lie within the sensitivity of ongoing searches $[45,47]$.

As mentioned in section 1, the recent Planck 2018 CMB data [49] puts tight constraints on Natural Inflation. The bi-axionic inflation studied here, while very roughly giving a Natural Inflation potential, can have significant differences at precision level that can be used to better agree with the data, as exemplified in [50]. We hope to further explore SUSY axionic inflation models in the future for the best fit to the precision data.

\section{A SUGRA preliminaries}

We write here the important SUGRA expressions relevant for the present paper. See [70] for review and further details.

For a general Kähler potential and superpotential for chiral superfields $\Phi_{i}$,

$$
K=K\left(\Phi_{i}, \bar{\Phi}_{i}\right), W=W\left(\Phi_{i}\right),
$$

the SUGRA scalar potential is

$$
V_{\text {scalar }}\left(\Phi_{i}, \bar{\Phi}_{i}\right)=e^{K}\left[K_{\Phi_{i} \bar{\Phi}_{j}}^{-1}\left(D_{\Phi_{i}} W\right)\left(D_{\bar{\Phi}_{j}} \bar{W}\right)-3 W \bar{W}\right]
$$


with subscripts referring to the respective partial derivatives, and with

$$
D_{\Phi_{i}} W \equiv W_{\Phi_{i}}+K_{\Phi_{i}} W
$$

$\left\langle D_{\Phi_{i}} W\right\rangle$ is the SUSY order parameter for each of the superfields $\Phi_{i}$. If there exists spontaneous breaking of SUSY in a model, it gives rise to a massless Goldstino,

$$
\psi_{\text {Goldstino }} \propto\left\langle D_{\Phi_{i}} W\right\rangle \psi_{\Phi_{i}}
$$

where $\psi_{\Phi_{i}}$ are fermions in the superfields $\Phi_{i}$. The Goldstino is further "eaten" by the gravitino which then becomes massive. This is called the "super-Higgs mechanism".

\section{Acknowledgments}

The authors would like to thank Soubhik Kumar, Marco Peloso and Jesse Thaler for helpful discussions. This research was supported in part by the NSF under Grant No. PHY-1620074 and by the Maryland Center for Fundamental Physics (MCFP).

Open Access. This article is distributed under the terms of the Creative Commons Attribution License (CC-BY 4.0), which permits any use, distribution and reproduction in any medium, provided the original author(s) and source are credited.

\section{References}

[1] D. Baumann, Inflation, in proceedings of the Theoretical Advanced Study Institute in Elementary Particle Physics: Physics of the Large and the Small (TASI 2009), Boulder, Colorado, U.S.A., 1-26 June 2009, pp. 523-686 [https://doi.org/10.1142/9789814327183_0010] [arXiv:0907.5424] [INSPIRE].

[2] K. Freese, J.A. Frieman and A.V. Olinto, Natural inflation with pseudo - Nambu-Goldstone bosons, Phys. Rev. Lett. 65 (1990) 3233 [inSPIRE].

[3] Planck collaboration, Planck 2015 results. XX. Constraints on inflation, Astron. Astrophys. 594 (2016) A20 [arXiv:1502.02114] [INSPIRE].

[4] D.H. Lyth, What would we learn by detecting a gravitational wave signal in the cosmic microwave background anisotropy?, Phys. Rev. Lett. 78 (1997) 1861 [hep-ph/9606387] [INSPIRE].

[5] R. Kallosh, A.D. Linde, D.A. Linde and L. Susskind, Gravity and global symmetries, Phys. Rev. D 52 (1995) 912 [hep-th/9502069] [INSPIRE].

[6] T. Banks and N. Seiberg, Symmetries and Strings in Field Theory and Gravity, Phys. Rev. D 83 (2011) 084019 [arXiv: 1011.5120] [INSPIRE].

[7] D. Harlow and H. Ooguri, Constraints on Symmetries from Holography, Phys. Rev. Lett. 122 (2019) 191601 [arXiv:1810.05337] [INSPIRE].

[8] J.E. Kim, H.P. Nilles and M. Peloso, Completing natural inflation, JCAP 01 (2005) 005 [hep-ph/0409138] [INSPIRE].

[9] N. Arkani-Hamed, H.-C. Cheng, P. Creminelli and L. Randall, Extra natural inflation, Phys. Rev. Lett. 90 (2003) 221302 [hep-th/0301218] [INSPIRE]. 
[10] Y. Bai and B.A. Stefanek, Natural millicharged inflation, Phys. Rev. D 91 (2015) 096012 [arXiv: 1405.6720] [INSPIRE].

[11] R. Contino, Y. Nomura and A. Pomarol, Higgs as a holographic pseudoGoldstone boson, Nucl. Phys. B 671 (2003) 148 [hep-ph/0306259] [InSPIRE].

[12] Y. Hosotani, Dynamical Gauge Symmetry Breaking as the Casimir Effect, Phys. Lett. B 129 (1983) 193 [INSPIRE].

[13] A. de la Fuente, P. Saraswat and R. Sundrum, Natural Inflation and Quantum Gravity, Phys. Rev. Lett. 114 (2015) 151303 [arXiv: 1412.3457] [INSPIRE].

[14] N. Arkani-Hamed, L. Motl, A. Nicolis and C. Vafa, The String landscape, black holes and gravity as the weakest force, JHEP 06 (2007) 060 [hep-th/0601001] [INSPIRE].

[15] M. Czerny, T. Higaki and F. Takahashi, Multi-Natural Inflation in Supergravity and BICEP2, Phys. Lett. B 734 (2014) 167 [arXiv:1403.5883] [INSPIRE].

[16] X. Gao, T. Li and P. Shukla, Combining Universal and Odd RR Axions for Aligned Natural Inflation, JCAP 10 (2014) 048 [arXiv:1406.0341] [INSPIRE].

[17] C. Long, L. McAllister and P. McGuirk, Aligned Natural Inflation in String Theory, Phys. Rev. D 90 (2014) 023501 [arXiv:1404.7852] [INSPIRE].

[18] T. Ali, S.S. Haque and V. Jejjala, Natural Inflation from Near Alignment in Heterotic String Theory, Phys. Rev. D 91 (2015) 083516 [arXiv:1410.4660] [INSPIRE].

[19] I. Ben-Dayan, F.G. Pedro and A. Westphal, Towards Natural Inflation in String Theory, Phys. Rev. D 92 (2015) 023515 [arXiv:1407.2562] [INSPIRE].

[20] E. Palti, On Natural Inflation and Moduli Stabilisation in String Theory, JHEP 10 (2015) 188 [arXiv: 1508.00009] [INSPIRE].

[21] R. Kappl, H.P. Nilles and M.W. Winkler, Natural Inflation and Low Energy Supersymmetry, Phys. Lett. B 746 (2015) 15 [arXiv:1503.01777] [INSPIRE].

[22] R. Kallosh and A.D. Linde, Landscape, the scale of SUSY breaking and inflation, JHEP 12 (2004) 004 [hep-th/0411011] [INSPIRE].

[23] T. He, S. Kachru and A. Westphal, Gravity waves and the LHC: Towards high-scale inflation with low-energy SUSY, JHEP 06 (2010) 065 [arXiv: 1003.4265] [INSPIRE].

[24] T. Kobayashi and M. Sakai, Inflation, moduli (de)stabilization and supersymmetry breaking, JHEP 04 (2011) 121 [arXiv: 1012.2187] [INSPIRE].

[25] S. Antusch, K. Dutta and S. Halter, Combining High-scale Inflation with Low-energy SUSY, JHEP 03 (2012) 105 [arXiv: 1112.4488] [INSPIRE].

[26] Y. Yamada, Instant uplifted inflation: A solution for a tension between inflation and SUSY breaking scale, JHEP 07 (2013) 039 [arXiv:1211.1455] [INSPIRE].

[27] M. Czerny, T. Higaki and F. Takahashi, Multi-Natural Inflation in Supergravity, JHEP 05 (2014) 144 [arXiv:1403.0410] [INSPIRE].

[28] W. Buchmüller, E. Dudas, L. Heurtier, A. Westphal, C. Wieck and M.W. Winkler, Challenges for Large-Field Inflation and Moduli Stabilization, JHEP 04 (2015) 058 [arXiv: 1501.05812] [INSPIRE].

[29] X. Chen and Y. Wang, Large non-Gaussianities with Intermediate Shapes from Quasi-Single Field Inflation, Phys. Rev. D 81 (2010) 063511 [arXiv:0909.0496] [INSPIRE]. 
[30] X. Chen and Y. Wang, Quasi-Single Field Inflation and Non-Gaussianities, JCAP 04 (2010) 027 [arXiv:0911.3380] [INSPIRE].

[31] X. Chen, Primordial Non-Gaussianities from Inflation Models, Adv. Astron. 2010 (2010) 638979 [arXiv: 1002.1416] [INSPIRE].

[32] D. Baumann and D. Green, Signatures of Supersymmetry from the Early Universe, Phys. Rev. D 85 (2012) 103520 [arXiv:1109.0292] [INSPIRE].

[33] V. Assassi, D. Baumann and D. Green, On Soft Limits of Inflationary Correlation Functions, JCAP 11 (2012) 047 [arXiv:1204.4207] [INSPIRE].

[34] X. Chen and Y. Wang, Quasi-Single Field Inflation with Large Mass, JCAP 09 (2012) 021 [arXiv: 1205.0160] [INSPIRE].

[35] S. Pi and M. Sasaki, Curvature Perturbation Spectrum in Two-field Inflation with a Turning Trajectory, JCAP 10 (2012) 051 [arXiv: 1205.0161] [INSPIRE].

[36] T. Noumi, M. Yamaguchi and D. Yokoyama, Effective field theory approach to quasi-single field inflation and effects of heavy fields, JHEP 06 (2013) 051 [arXiv:1211.1624] [InSPIRE].

[37] N. Arkani-Hamed and J. Maldacena, Cosmological Collider Physics, arXiv:1503.08043 [INSPIRE].

[38] E. Dimastrogiovanni, M. Fasiello and M. Kamionkowski, Imprints of Massive Primordial Fields on Large-Scale Structure, JCAP 02 (2016) 017 [arXiv:1504.05993] [INSPIRE].

[39] S. Kumar and R. Sundrum, Heavy-Lifting of Gauge Theories By Cosmic Inflation, JHEP 05 (2018) 011 [arXiv : 1711.03988] [INSPIRE].

[40] X. Wang, B. Feng, M. Li, X.-L. Chen and X. Zhang, Natural inflation, Planck scale physics and oscillating primordial spectrum, Int. J. Mod. Phys. D 14 (2005) 1347 [astro-ph/0209242] [INSPIRE].

[41] C. Pahud, M. Kamionkowski and A.R. Liddle, Oscillations in the inflaton potential?, Phys. Rev. D 79 (2009) 083503 [arXiv:0807.0322] [INSPIRE].

[42] R. Flauger, L. McAllister, E. Pajer, A. Westphal and G. Xu, Oscillations in the CMB from Axion Monodromy Inflation, JCAP 06 (2010) 009 [arXiv:0907.2916] [INSPIRE].

[43] T. Kobayashi and F. Takahashi, Running Spectral Index from Inflation with Modulations, JCAP 01 (2011) 026 [arXiv: 1011.3988] [INSPIRE].

[44] R. Easther and R. Flauger, Planck Constraints on Monodromy Inflation, JCAP 02 (2014) 037 [arXiv: 1308.3736] [INSPIRE].

[45] R. Flauger, L. McAllister, E. Silverstein and A. Westphal, Drifting Oscillations in Axion Monodromy, JCAP 10 (2017) 055 [arXiv: 1412.1814] [INSPIRE].

[46] T. Higaki and F. Takahashi, Axion Landscape and Natural Inflation, Phys. Lett. B 744 (2015) 153 [arXiv:1409.8409] [INSPIRE].

[47] K. Choi and H. Kim, Aligned natural inflation with modulations, Phys. Lett. B 759 (2016) 520 [arXiv: 1511.07201] [INSPIRE].

[48] L.C. Price, Power spectrum oscillations from Planck-suppressed operators in effective field theory motivated monodromy inflation, Phys. Rev. D 92 (2015) 103507 [arXiv:1507.08360] [INSPIRE]. 
[49] Planck collaboration, Planck 2018 results. X. Constraints on inflation, arXiv:1807.06211 [INSPIRE].

[50] M. Peloso and C. Unal, Trajectories with suppressed tensor-to-scalar ratio in Aligned Natural Inflation, JCAP 06 (2015) 040 [arXiv: 1504.02784] [INSPIRE].

[51] M. Kawasaki, M. Yamaguchi and T. Yanagida, Natural chaotic inflation in supergravity, Phys. Rev. Lett. 85 (2000) 3572 [hep-ph/0004243] [INSPIRE].

[52] R. Kallosh, A.D. Linde and T. Rube, General inflaton potentials in supergravity, Phys. Rev. D 83 (2011) 043507 [arXiv: 1011.5945] [InSPIRE].

[53] A. Achucarro, S. Mooij, P. Ortiz and M. Postma, Sgoldstino inflation, JCAP 08 (2012) 013 [arXiv: 1203.1907] [INSPIRE].

[54] S. Ferrara and D. Roest, General sGoldstino Inflation, JCAP 10 (2016) 038 [arXiv: 1608.03709] [INSPIRE].

[55] S.V. Ketov and T. Terada, Generic Scalar Potentials for Inflation in Supergravity with a Single Chiral Superfield, JHEP 12 (2014) 062 [arXiv:1408.6524] [InSPIRE].

[56] L. Álvarez-Gaumé, C. Gomez and R. Jimenez, A Minimal Inflation Scenario, JCAP 03 (2011) 027 [arXiv: 1101.4948] [INSPIRE].

[57] J. Polonyi, Generalization of the Massive Scalar Multiplet Coupling to the Supergravity, Central Research Institute for Physics, Budapest Hungary (1977) [KFKI-77-93] [https://doi.org/10.13140/RG.2.1.4621.4884].

[58] N. Arkani-Hamed, T. Gregoire and J.G. Wacker, Higher dimensional supersymmetry in 4 - D superspace, JHEP 03 (2002) 055 [hep-th/0101233] [INSPIRE].

[59] R. Sundrum, TASI 2004 lectures: To the fifth dimension and back, in proceedings of the Theoretical Advanced Study Institute in Elementary Particle Physics: Many Dimensions of String Theory (TASI 2005), Boulder, Colorado, U.S.A., 5 June-1 July 2005, pp. 585-630 [hep-th/0508134] [INSPIRE].

[60] E. Dudas and C. Wieck, Moduli backreaction and supersymmetry breaking in string-inspired inflation models, JHEP 10 (2015) 062 [arXiv: 1506.01253] [INSPIRE].

[61] M.R. Douglas and S. Kachru, Flux compactification, Rev. Mod. Phys. 79 (2007) 733 [hep-th/0610102] [INSPIRE].

[62] S. Weinberg, The Cosmological Constant Problem, Rev. Mod. Phys. 61 (1989) 1 [InSPIRE].

[63] R. Bousso, TASI Lectures on the Cosmological Constant, Gen. Rel. Grav. 40 (2008) 607 [arXiv:0708.4231] [INSPIRE].

[64] Y. Kahn, D.A. Roberts and J. Thaler, The goldstone and goldstino of supersymmetric inflation, JHEP 10 (2015) 001 [arXiv:1504.05958] [INSPIRE].

[65] S. Ferrara, R. Kallosh and J. Thaler, Cosmology with orthogonal nilpotent superfields, Phys. Rev. D 93 (2016) 043516 [arXiv: 1512.00545] [INSPIRE].

[66] L.V. Delacretaz, V. Gorbenko and L. Senatore, The Supersymmetric Effective Field Theory of Inflation, JHEP 03 (2017) 063 [arXiv: 1610.04227] [INSPIRE].

[67] A. Loeb and M. Zaldarriaga, Measuring the small - scale power spectrum of cosmic density fluctuations through $21 \mathrm{~cm}$ tomography prior to the epoch of structure formation, Phys. Rev. Lett. 92 (2004) 211301 [astro-ph/0312134] [INSPIRE]. 
[68] J.B. Muñoz, Y. Ali-Haïmoud and M. Kamionkowski, Primordial non-Gaussianity from the bispectrum of 21-cm fluctuations in the dark ages, Phys. Rev. D 92 (2015) 083508 [arXiv: 1506.04152] [INSPIRE].

[69] P.D. Meerburg, M. Münchmeyer, J.B. Muñoz and X. Chen, Prospects for Cosmological Collider Physics, JCAP 03 (2017) 050 [arXiv: 1610.06559] [INSPIRE].

[70] J. Wess and J. Bagger, Supersymmetry and supergravity, Princeton University Press, Princeton NJ U.S.A. (1992). 